\title{
The shape of CMB temperature and polarization peaks on the sphere
}

\author{
A. Marcos-Caballero, ${ }^{a, b}$ R. Fernández-Cobos, ${ }^{a}$ \\ E. Martínez-González ${ }^{a}$ and P. Vielva ${ }^{a}$ \\ ${ }^{a}$ Instituto de Física de Cantabria, CSIC-Universidad de Cantabria, \\ Avda. de los Castros s/n, 39005 Santander, Spain. \\ ${ }^{b}$ Dpto. de Física Moderna, Universidad de Cantabria, \\ Avda. los Castros s/n, 39005 Santander, Spain. \\ E-mail: marcos@ifca.unican.es, cobos@ifca.unican.es, martinez@ifca.unican.es, \\ vielva@ifca.unican.es
}

\begin{abstract}
We present a theoretical study of CMB temperature peaks, including its effect over the polarization field, and allowing nonzero eccentricity. The formalism is developed in harmonic space and using the covariant derivative on the sphere, which guarantees that the expressions obtained are completely valid at large scales (i.e., no flat approximation). The expected patterns induced by the peak, either in temperature or polarization, are calculated, as well as their covariances. It is found that the eccentricity introduces a quadrupolar dependence in the peak shape, which is proportional to a complex bias parameter $b_{\epsilon}$, characterizing the peak asymmetry and orientation. In addition, the one-point statistics of the variables defining the peak on the sphere is reviewed, finding some differences with respect to the flat case for large peaks. Finally, we present a mechanism to simulate constrained CMB maps with a particular peak on the field, which is an interesting tool for analysing the statistical properties of the peaks present in the data.
\end{abstract}




\section{Contents}

1 Introduction 1

2 Derivatives of a scalar field on the sphere 3

3 Uncorrelating the peak variables 4

4 Extrema statistics $\quad 6$

5 Multipolar profiles $\quad 8$

5.1 Profiles in harmonic space $\quad 9$

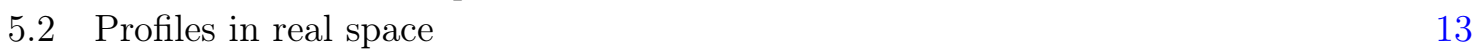

5.3 Bias discussion 16

6 Covariance of the multipolar profiles 19

$\begin{array}{lll}7 & \text { Physical interpretation of the peak patterns } & 21\end{array}$

8 Peak simulations $\quad 23$

9 Conclusions $\quad 24$

$\begin{array}{ll}\text { A Covariant derivatives on the sphere } & 26\end{array}$

$\begin{array}{ll}\text { B Peak degrees of freedom } & 28\end{array}$

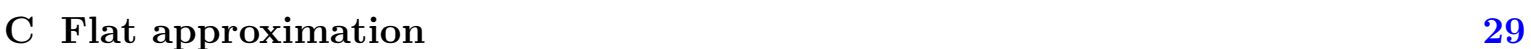

\section{Introduction}

The cosmic microwave background (CMB) radiation is one of the most important sources of cosmological information. In particular, the statistical properties of the CMB fluctuations are essential to understand the primordial Universe. In order to explain the observations, a phase of inflationary expansion in the early Universe has been postulated. Within the standard frame, this inflation mechanism also generates the initial matter perturbations which are the seeds of the cosmic structures observed nowadays. It is believed that the initial perturbations generated by the standard inflationary models are nearly Gaussian. For this reason, the 2point correlation functions of the temperature and polarization CMB anisotropies have most of the cosmological information which can be obtained from the primordial fluctuations. The temperature angular power spectrum of the CMB has been recently determined by the Planck mission with high accuracy [1]. Regarding the primordial CMB polarization, only the gradient part of the polarization field ( $E$-mode) has been detected up to $\ell \sim 2000$. Although the effect of the gravitational lensing on the curl of the polarization field ( $B$-mode) has been observed $[2,3]$, there is still no evidence of the primordial $B$-mode induced by the tensor perturbations $[4,5])$. On the whole, the agreement of the CMB data with the cosmological standard model is high [6]. However, there are several anomalies at large scales which are still unexplained [7]. 
One of the alternative observables which can be used to study the perturbations is the statistical properties of peaks. In the case of the matter field, it is important to understand the properties of overdensity peaks because that is where the collapse of structures takes place. In a seminal work [8], the statistics of peaks for Gaussian fields in three dimensions is developed. There are several aspects of peaks which can be analysed, for instance, the number of peaks, the peak shape or their correlation function. One important result from peak theory is that the peak correlation function is related to the underlying matter distribution through a non-local bias [9]. The understanding of the peak correlation function and its bias relation to the matter field is also important to study the baryon acoustic oscillations [10].

In order to study the CMB temperature extrema, the three-dimensional formalism of peaks was later particularised in [11] to the case of scalar fields on the sphere (see also [12]). However, a full analysis including polarization is needed for a complete understanding of the CMB fluctuations. The radial profiles of the Stokes parameters were described in [13]. Nevertheless, these profiles are calculated using the small-angle limit and the peaks are considered spherically symmetric. Recently, an analysis of the CMB temperature and polarization Planck data, including peak eccentricity, has been published [7]. The studies in [13] and [7] based on the stacking of peaks do not reveal significant deviations from the standard model, except a shift in the temperature profile which could be associated to the power deficit at large scales. Non-standard scenarios including parity violations [14] or cosmological birefringence [15] can also be tested using the stacking of temperature peaks in polarization.

In this paper, we present a comprehensive study of the CMB peaks on the sphere including polarization and allowing different eccentricities. The derivation followed in this work is based on the spherical harmonic coefficients, instead of the real space. This allows to obtain expressions which are completely valid at large scales, where the flat approximation breaks. In addition, the formalism in harmonic space opens the possibility of generating constrained CMB simulations with a peak at some point of the sphere with the desired characteristics. Besides the peak shapes, the extrema statistics is reviewed for the case of a Gaussian scalar field on the sphere. It is found that the probability and the number density of large peaks is modified with respect to the calculations in [11]. Finally, we notice that the approach addressed in this work is completely general and it can be applied to any scalar Gaussian field on the sphere, taking into account its correlation with any other scalar or spin-2 field.

This paper is organized as follows: in section 2, we introduce the covariant derivatives in terms of the spherical harmonic coefficients in order to define the peak degrees of freedom on the sphere. In section 3, it is explained the methodology used to separate the variables defining the peak from the rest of the Gaussian random field. Additionally, the statistics of extrema on the sphere is reviewed in section 4, finding some differences with respect to previous calculations. The shape of CMB peaks including polarization for different values of mean curvature and eccentricity is analysed in section 5, whilst its covariance is calculated in section 6. The expressions of the peak patterns are given in terms of the angular power spectra, which allows to calculate them in a simple way. The physical description of the peak profiles is discussed in section 7 . Furthermore, in section 8 , a way to simulate peaks on the sphere, which is one of the applications of the formalism developed in this work, is derived. Finally, the conclusions of the paper are presented in section 9. 


\section{Derivatives of a scalar field on the sphere}

A peak on the sphere is defined through its derivatives up to second order. In general, any field on the sphere can be expanded in terms of the spherical harmonics:

$$
T(\theta, \phi)=\sum_{\ell=0}^{\infty} a_{\ell m} Y_{\ell m}(\theta, \phi) .
$$

The first step in our analysis is to express the derivatives in terms of the spherical harmonic coefficients $a_{\ell m}$. For simplicity, we consider that the peak is located at the north pole. The value of the field at this point can be written in the following way:

$$
T=\sum_{\ell=0}^{\infty} \sqrt{\frac{2 \ell+1}{4 \pi}} a_{\ell 0} .
$$

In order to calculate derivatives on the sphere, we use the spin raising and lowering operators $\not \partial$ and $\not^{*}$, which are proportional to the covariant derivatives in the helicity basis (see appendix A for a more detailed description of the derivatives on the sphere). If we consider the local system of reference at any point of the sphere, then the derivatives with respect to the Cartesian coordinates correspond to the real and imaginary parts of the lowering operator, that is $\not \not^{*}=-\partial_{x}+i \partial_{y}$ (similarly, the spin raising operator verifies $\not \partial=-\partial_{x}-i \partial_{y}$ ). Here, we have assumed that the basis vectors $\mathbf{e}_{x}$ and $\mathbf{e}_{y}$ correspond to the vectors $\mathbf{e}_{\theta}$ and $\mathbf{e}_{\phi}$ of the spherical coordinate system, respectively. Thus, the first derivatives at this point are written as

$$
\partial^{*} T=\sum_{\ell=0}^{\infty} \sqrt{\frac{2 \ell+1}{4 \pi}} \sqrt{\frac{(\ell+1) !}{(\ell-1) !}} a_{\ell 1} .
$$

This quantity is a complex number whose real and imaginary parts correspond to the derivatives in each orthogonal direction at the north pole. Finally, the second derivatives are encoded in the Hessian matrix (see eq. (B.1)). It is convenient to separate the trace and the traceless parts of this matrix, because these two quantities transform in a different way under rotations. The trace corresponds to the Laplacian,

$$
\nabla^{2} T=\not \partial * \partial T=-\sum_{\ell=0}^{\infty} \sqrt{\frac{2 \ell+1}{4 \pi}} \frac{(\ell+1) !}{(\ell-1) !} a_{\ell 0},
$$

and the traceless part is given by

$$
\left.(\not)^{*}\right)^{2} T=\sum_{\ell=0}^{\infty} \sqrt{\frac{2 \ell+1}{4 \pi}} \sqrt{\frac{(\ell+2) !}{(\ell-2) !}} a_{\ell 2} .
$$

In the local system of reference, this operator is given by $\left(\not{ }^{*}\right)^{2}=\partial_{x}^{2}-\partial_{y}^{2}-i 2 \partial_{x} \partial_{y}$. Although this operator is a complex quantity, the imaginary part can be set to zero with a rotation of the $x y$ plane. Physically, this corresponds to choose the principal axes of the peak as the reference system. From the real part, it can be shown that the operator $\left(\not \not^{*}\right)^{2}$ represents a measure of the anisotropy at the centre of the peak.

The $a_{\ell m}$ coefficients are $m$-spin quantities under rotations of the $z$ axis. That is, if we rotate by an angle $\alpha$, the $a_{\ell m}$ coefficient transforms as $a_{\ell m} e^{i m \alpha}$. Looking at the expression 
of the field derivatives in terms of the spherical harmonic coefficients, it is possible to deduce that, whilst $T$ and $\nabla^{2} T$ are scalars, $\not \partial^{*} T$ is a vector and $\left(\not \not^{*}\right)^{2} T$ is a 2 -spin tensor. Since tensors with different rank are statistically independent under the assumption of isotropy, then only the scalars $T$ and $\nabla^{2} T$ are correlated, while $\not{ }^{*} T$ and $\left(\not \not^{*}\right)^{2} T$ are uncorrelated with the rest of the field derivatives.

For simplicity, we normalize the field derivatives in order to have unit variance:

$$
\begin{array}{cc}
\nu \equiv \frac{T}{\sigma_{\nu}}, & \kappa \equiv-\frac{\nabla^{2} T}{\sigma_{\kappa}}, \\
\eta \equiv \frac{\not \partial^{*} T}{\sigma_{\eta}}, & \epsilon \equiv \frac{\left.(\not)^{*}\right)^{2} T}{\sigma_{\epsilon}},
\end{array}
$$

where the expressions of the variances are given in the appendix B. These parameters denote the peak degrees of freedom throughout the paper. The parameter $\nu$ represents the peak height, whereas the normalized Laplacian $\kappa$ is the mean curvature of the peak. The parameter $\eta$ is a complex number whose components are the first derivatives at the peak location. Hereafter, we set $\eta=0$ in order to have a critical point. Finally, the value of $\epsilon$ gives information about the eccentricity of the peak. In particular, its modulus is proportional to the square of the eccentricity, and its phase is twice the orientation angle with respect to the reference system (see more details in appendix B).

Using eqs. (2.2), the peak variables can be expanded in terms of the spherical harmonic coefficients, which are normalized to have unit variance:

$$
\begin{array}{ll}
\nu=\sum_{\ell=0}^{\infty} \nu_{\ell} a_{\ell 0}, \quad \kappa=\sum_{\ell=0}^{\infty} \kappa_{\ell} a_{\ell 0}, \\
\eta=\sum_{\ell=0}^{\infty} \eta_{\ell} a_{\ell 1}, \quad \epsilon=\sum_{\ell=0}^{\infty} \epsilon_{\ell} a_{\ell 2},
\end{array}
$$

where the multipolar coefficients $\nu_{\ell}, \kappa_{\ell}, \eta_{\ell}$ and $\epsilon_{\ell}$ are defined in the appendix B (eqs. (B.4)).

\section{Uncorrelating the peak variables}

The aim of this section is to separate the peak degrees of freedom from the rest of the information of the field. For this purpose, we transform the $a_{\ell m}$ coefficients into a new set of variables containing the peak degrees of freedom $(\nu, \kappa, \eta$ and $\epsilon)$ and an ensemble of new variables $\hat{a}_{\ell m}$ without any peak information. The $\hat{a}_{\ell m}$ variables are defined for all values of $\ell$ except for four given multipoles $\ell_{\nu}, \ell_{\kappa}, \ell_{\eta}$ and $\ell_{\epsilon}$, in order to preserve the total number of degrees of freedom. ${ }^{1}$ We choose the variables $\hat{a}_{\ell m}$ such that its correlation with the peak variables vanishes, using an orthogonalization process. For convenience, we normalize the $a_{\ell m}$ coefficients such that they have unit variance. The change of variables is given by:

$$
\begin{gathered}
\hat{a}_{\ell 0}=a_{\ell 0}-\left(\begin{array}{ll}
a_{\ell_{\nu} 0} & a_{\ell_{\kappa} 0}
\end{array}\right) P^{-1}\left(\begin{array}{c}
\nu_{\ell} \\
\kappa_{\ell}
\end{array}\right) \quad\left(\ell \neq \ell_{\nu}, \ell_{\kappa}\right), \\
\hat{a}_{\ell 1}=a_{\ell 1}-a_{\ell_{\eta}} \frac{\eta_{\ell}}{\eta_{\ell_{\eta}}} \quad\left(\ell \neq \ell_{\eta}\right),
\end{gathered}
$$

\footnotetext{
${ }^{1}$ In principle, the multipoles $\ell_{\nu}, \ell_{\kappa}, \ell_{\eta}$ and $\ell_{\epsilon}$ are chosen arbitrarily with the condition that $\nu_{\ell_{\nu}}, \kappa_{\ell_{\kappa}}, \eta_{\ell_{\eta}}, \epsilon_{\ell_{\epsilon}} \neq 0$, such that the change of variables is not singular.
} 


$$
\begin{gathered}
\hat{a}_{\ell 2}=a_{\ell 2}-a_{\ell_{\epsilon} 2} \frac{\epsilon_{\ell}}{\epsilon_{\ell_{\epsilon}}} \quad\left(\ell \neq \ell_{\epsilon}\right), \\
\hat{a}_{\ell m}=a_{\ell m} \quad(m>2) .
\end{gathered}
$$

The peak variables only affect to the multipoles $m=0,1,2$, and therefore the $a_{\ell m}$ coefficients with $m>2$ remain unchanged. The matrix $P$ in eq. (3.1a) is the pivot matrix given by

$$
P=\left(\begin{array}{ll}
\nu_{\ell_{\nu}} & \nu_{\ell_{\kappa}} \\
\kappa_{\ell_{\nu}} & \kappa_{\ell_{\kappa}}
\end{array}\right) .
$$

Notice that the variables $\hat{a}_{\ell m}$ are not the coefficients of the standard spherical harmonics expansion. The inverse relations between $a_{\ell m}$ and $\hat{a}_{\ell m}$ are calculated from eqs. (3.1) with a little bit of algebra:

$$
\begin{aligned}
& a_{\ell 0}=\hat{a}_{\ell 0}+\left(\nu_{\ell} \kappa_{\ell}\right) \Sigma^{-1}\left[\left(\begin{array}{c}
\nu \\
\kappa
\end{array}\right)-\sum_{\ell^{\prime}=0}^{\infty}\left(\begin{array}{c}
\nu_{\ell^{\prime}} \\
\kappa_{\ell^{\prime}}
\end{array}\right) \hat{a}_{\ell^{\prime} 0}\right], \\
& a_{\ell 1}=\hat{a}_{\ell 1}+\eta_{\ell}\left(\eta-\sum_{\ell^{\prime}=0}^{\infty} \eta_{\ell^{\prime}} \hat{a}_{\ell^{\prime} 1}\right) \\
& a_{\ell 2}=\hat{a}_{\ell 2}+\epsilon_{\ell}\left(\epsilon-\sum_{\ell^{\prime}=0}^{\infty} \epsilon_{\ell^{\prime}} \hat{a}_{\ell^{\prime} 2}\right), \\
& a_{\ell m}=\hat{a}_{\ell m} \quad(m>2) .
\end{aligned}
$$

For simplicity, we have assumed that these equations are valid for all $\ell$ with the prescription that the pivot coefficients $\hat{a}_{\ell_{\nu} 0}, \hat{a}_{\ell_{\kappa} 0}, \hat{a}_{\ell_{\eta} 1}, \hat{a}_{\ell_{\epsilon} 2}$ are zero. The matrix $\Sigma$ in eq. (3.3a) is the covariance matrix between $\nu$ and $\kappa$. As the peak variables are uncorrelated with the $\hat{a}_{\ell m}$ coefficients, it is possible to put constraints in $\nu, \kappa, \eta$ and $\epsilon$ without affecting the rest of the degrees of freedom of the temperature, given by the variables $\hat{a}_{\ell m}$. Once the peak constraints are imposed, the original $a_{\ell m}$ coefficients are recovered using the inverse relation. Notice that this inversion process is analytical and therefore no numerical inversion is needed.

In addition to the temperature field, we can also consider the $E$ and $B$ polarization fields. Although the peak selection is still done in $T$, the $E$ and $B$-modes will be affected due to the corresponding correlation between both fields and $T$. Once we have specified the peak conditions on the temperature, we need to know what is the conditional probability of $E$ and $B$, in order to calculate their statistical properties. Although the primordial fluctuations do not introduce correlation between the $B$-mode and the scalar fields, there could be different physical effects which break the parity invariance of the field and lead to the $T B$ and $E B$ correlations $[14,15]$. Within the formalism established in this paper, we consider the general case where these correlations are non-zero. If the distribution of the temperature and polarization fields is Gaussian, then the conditional probability of $E$ and $B$ given $T$ is a bivariate Gaussian with the following mean values and covariance:

$$
\begin{gathered}
\left\langle e_{\ell m}\right\rangle=\frac{C_{\ell}^{T E}}{\sqrt{C_{\ell}^{T T}}} a_{\ell m}, \quad\left\langle b_{\ell m}\right\rangle=\frac{C_{\ell}^{T B}}{\sqrt{C_{\ell}^{T T}}} a_{\ell m}, \\
\mathbf{C}=\left(\begin{array}{c}
C_{\ell}^{E E} C_{\ell}^{E B} \\
C_{\ell}^{E B} C_{\ell}^{B B}
\end{array}\right)-\frac{1}{C_{\ell}^{T T}}\left(\begin{array}{cc}
\left(C_{\ell}^{T E}\right)^{2} & C_{\ell}^{T E} C_{\ell}^{T B} \\
C_{\ell}^{T E} C_{\ell}^{T B} & \left(C_{\ell}^{T E}\right)^{2}
\end{array}\right),
\end{gathered}
$$


where $e_{\ell m}$ and $b_{\ell m}$ are the spherical harmonic coefficients of $E$ and $B$ respectively. The mean values of the polarization modes are affected by the temperature field, which is described by the $a_{\ell m}$ coefficients in these equations. The constraints on the temperature due to the peak induce a non-zero pattern in the polarization fields. This fact is used in section 5 to calculate the shape of peaks in polarization.

\section{Extrema statistics}

In this section we show how to select minima or maxima using the peak variables $\nu, \kappa, \eta$ and $\epsilon$. In order to have a critical point, the only requirement is to fix the first derivatives to zero, that is, $\eta=0$. In addition, if we want to have an extremum, additional constraints in the mean curvature $\kappa$ and in the eccentricity $\epsilon$ are needed. In particular, we ensure that the critical point is an extremum by requiring that the eigenvalues of the Hessian matrix have the same sign. This is done by imposing that $|\epsilon| \leq \sqrt{a}|\kappa|$, where $a=\sigma_{\kappa}^{2} / \sigma_{\epsilon}^{2}$ is the ratio of the variance of the Laplacian $\nabla^{2} T$ and that of $\left(\not \partial^{*}\right)^{2} T$. Whether that extremum is a minimum or maximum depends on the sign of the curvature. If $\kappa>0$, the field will have a maximum, and, if $\kappa<0$, a minimum (in the case of $\kappa=0$, the point would be flat up to second order, but the probability of this is zero). These extremum constraints can be imposed by considering the probability of the peak degrees of freedom:

$$
P(\nu, \kappa, \epsilon) \mathrm{d} \nu \mathrm{d} \kappa \mathrm{d}^{2} \epsilon=\frac{2|\epsilon|}{2 \pi \sqrt{\left(1-\rho^{2}\right)}} \exp \left[-\frac{\nu^{2}-2 \rho \nu \kappa+\kappa^{2}}{2\left(1-\rho^{2}\right)}-|\epsilon|^{2}\right] \mathrm{d} \nu \mathrm{d} \kappa \mathrm{d}|\epsilon| \frac{\mathrm{d} \alpha}{\pi}
$$

where the eccentricity is given by $\epsilon=|\epsilon| e^{i 2 \alpha}$, that is, $|\epsilon|$ is the modulus and $\alpha$ is the orientation of the ellipse. Notice that the peak height and the curvature are not independent, with a joint probability given by a multivariate Gaussian, where $\rho$ is the correlation. It is possible to write the correlation as a function of the field variances: $\rho=\sigma_{\eta}^{2} / \sigma_{\nu} \sigma_{\kappa}$. As the eccentricity is a Gaussian complex number, its modulus follows the Rayleigh distribution, and the orientation angle $\alpha$ is distributed uniformly in the interval $[0, \pi]$.

It is possible to calculate the number density of peaks from the probability density in eq. (4.1). The density of peaks depends on the particular size of the peak, in addition to its probability. For instance, the number of big spots is suppressed because they occupy an area larger than the small ones. Hence, it is expected that small spots are more abundant than large ones. The spot size dependence is introduced through the determinant of the Hessian matrix, which is proportional to the inverse of the square of the spot size. The number density of peaks is given by

$$
n(\nu, \kappa, \epsilon) \mathrm{d} \nu \mathrm{d} \kappa \mathrm{d}^{2} \epsilon=\frac{1}{2 \pi \theta_{*}^{2}}\left(a \kappa^{2}-|\epsilon|^{2}\right) P(\nu, \kappa, \epsilon) \mathrm{d} \nu \mathrm{d} \kappa \mathrm{d}^{2} \epsilon
$$

where $\theta_{*}^{2}=2 \sigma_{\eta}^{2} / \sigma_{\epsilon}^{2}$. This expression differs from the one in [11] in the $a$ parameter, but we recover it when $a \approx 1$. It is possible to show that there exists the constraint relation $a=1+\theta_{*}^{2}$ between both parameters. In the small-scale limit, it is possible to consider that $\theta_{*} \ll 1$, and then $a \approx 1$. Nevertheless, this limit is not valid if the sample is dominated by large spots ( see figure 1).

The expected total number of extrema on the sphere is obtained by integrating eq. (4.2) over all possible values of $\nu$ and $\kappa$. However, the integration over the eccentricity $\epsilon$ must be 

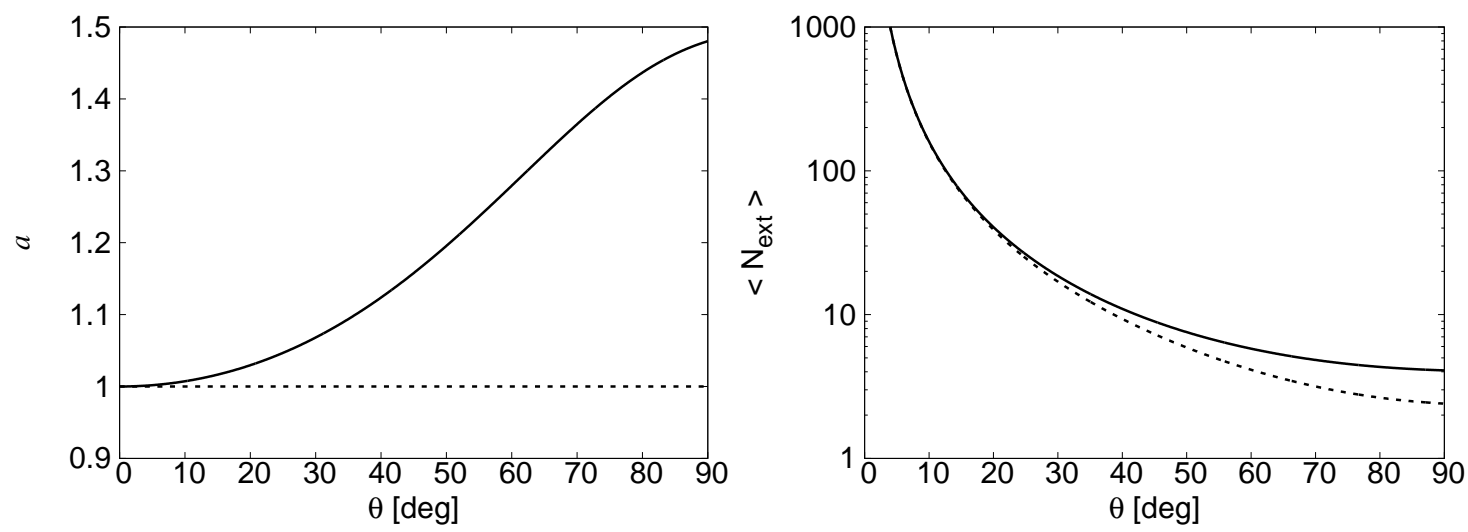

Figure 1. The parameter $a=\sigma_{\kappa}^{2} / \sigma_{\epsilon}^{2}$ and the expected number of extrema are depicted as a function of the angluar size of the peak. The solid lines represent the calculation for the sphere, whereas the dashed lines correspond to the flat approximation. In order to select peaks with a given size, the temperature field is filtered by a Gaussian whose FWHM corresponds to the peak scale considered. Notice that in the case of large peaks, for which the flat approximation breaks, it is necessary to use the sterographic projection to relate the FWHM of the Gaussian and the angular size of the peak $(R=2 \tan \theta / 2)$.

done in the region where $|\epsilon| \leq \sqrt{a}|\kappa|$, in order to guarantee that the point is an extremum. The expected total number of extrema is

$$
\left\langle N_{\text {ext }}\right\rangle=2\left(1+\frac{1}{\theta_{*}^{2} \sqrt{3+2 \theta_{*}^{2}}}\right) .
$$

This number only depends on the value of $\theta_{*}$. When $\theta_{*}$ is small, the number of extrema is proportional to $\theta_{*}^{-2}$. In this case, we obtain the result in [11]. But we need to consider the exact formula when the number of peaks is small, or equivalently, when the field is dominated by large peaks. In figure 1, the expected number of extrema is represented as a function of the peak size, where it is possible to see that the flat approximation breaks for peaks larger than $30^{\circ}$. In practice, there are only several of such large peaks on the sphere, and therefore the cosmic variance is not significantly reduced by performing a stacking analysis. However, the study of particularly large peaks is still useful to test the properties of the CMB at large scales.

As concrete examples of eq. 4.3, it is possible to check this expression analytically for dipolar and quadrupolar patterns. In the case of the dipole, we assume that $C_{1}^{T T} \neq 0$ and $C_{\ell}^{T T}=0$ for all $\ell \neq 1$. Therefore, the field will have a dipole with random orientation and amplitude. The small-scale limit cannot be taken in this case, since $\theta_{*}^{2}=\infty$. The number of extrema in a dipolar pattern is always 2, independently of the randomness of the field. This fact agrees with the prediction from eq. (4.3) for a random dipole, which is $\left\langle N_{\text {ext }}\right\rangle=2$. Repeating the same reasoning for a random quadrupole $\left(C_{2}^{T T} \neq 0\right.$ and $C_{\ell}^{T T}=0$, for $\left.\ell \neq 2\right)$, we find that $\left\langle N_{\text {ext }}\right\rangle=4$, as expected from a quadrupolar pattern which always has 2 maxima and 2 minima. In general, from eq. (4.3) follows that $\left\langle N_{\text {ext }}\right\rangle \geq 2$, reflecting the fact that there will be always one minimum and one maximum in the sphere at least. This is a consequence of the extreme value theorem applied to the sphere.

We finish this section commenting that there are two ways of assigning probabilities to the peak variables, depending on the physical problem we are addressing. If we are interested 
in studying the statistical properties of a single peak on the sphere, then we have to use the probability in eq. (4.1). This probability gives the distribution of the peak variables in a single point, independently of any other location on the sphere. On the other hand, sometimes it is useful to sum over a given population of peaks in order to enhance the signal we want to measure. The distribution of the peak variables in this stacking-like procedure is different from the one-point distribution. In this case, the correct way to assign probabilities is given by the number density in eq. (4.2). The peak variables of the stacked points are distributed on the sphere following the number density, instead of the one-point probability of the peak variables.

\section{$5 \quad$ Multipolar profiles}

The expected 2-dimensional shape of peaks on the sphere depends on how the peak variables are constrained. Indeed, if the peak degrees of freedom are randomly distributed without any additional constraint, then the expected pattern is zero. It is possible to see the peak shape as an effect of a bias in the peak variables. For instance, if we impose a threshold for the peak height $\nu$, then the randomness of the field is broken and the value of $\langle\nu\rangle$ is different from zero. This bias in the expected value of the peak height generates a non-trivial pattern on the sphere. In this section we only consider the peak height $\nu$, the mean curvature $\kappa$ and the eccentricity $\epsilon$ as the peak degrees of freedom, because the first order derivatives are fixed to zero $(\eta=0)$ by definition of a peak.

The fact that the expected value of the eccentricity $\langle\epsilon\rangle$ could be biased introduces a $\phi$ dependence in the peak pattern on the sphere. In order to take into account these angular dependence, we expand a generic field on the sphere $X(\theta, \phi)$ in the following way:

$$
X(\theta, \phi)=\sum_{m=-\infty}^{\infty} X_{m}(\theta) e^{i m \phi} .
$$

The profiles $X_{m}(\theta)$ represent contributions to the peak with different rotational symmetry. Since the field $X$ is real, it is satisfied that $X_{m}(\theta)^{*}=X_{-m}(\theta)$, where the number $m$ is the spin of the profile. The fact that peaks are determined through their derivatives up to second order implies that the profiles with spin $m>2$ vanish. The dipolar profile with $m=1$ is also zero because the first derivatives are zero by definition. Only the scalar $(m=0)$ and quadrupolar $(m=2)$ profiles contribute to this expansion.

The inverse transform of eq. (5.1) is

$$
X_{m}(\theta)=\frac{1}{2 \pi} \int \mathrm{d} \phi X(\theta, \phi) e^{-i m \phi} .
$$

In particular, the scalar profile $X_{0}(\theta)$ is the $\phi$-average of the field, that is, the standard profile when spherical symmetry is assumed. The quadrupolar profile $X_{2}(\theta)$ is a correction term due to the asymmetry introduced by the eccentricity of the peak. It is useful to write the multipolar profiles by using the associated Legendre polynomials:

$$
X_{m}(\theta)=\sum_{\ell=m}^{\infty} \sqrt{\frac{2 \ell+1}{4 \pi}} \sqrt{\frac{(\ell-m) !}{(\ell+m) !}} a_{\ell m}^{X} P_{\ell}^{m}(\cos \theta) .
$$

In the particular case when the peak is located in the north pole, the coefficients $a_{\ell m}^{X}$ in this expansion coincide with the spherical harmonics coefficients of the field $X$. 


\subsection{Profiles in harmonic space}

In this subsection, the multipolar profiles for the $\mathrm{CMB}$ temperature and polarization are calculated in harmonic space. For simplicity, it is convenient to use the Stokes parameters in polar coordinates with the origin at the centre of the peak. These polar parameters $Q_{r}$ and $U_{r}$ are a rotated version of the standard $Q$ and $U$ ones (see [16]). The $Q_{r}$ field represents radial or tangential polarization patterns around the spot. If the sign of $Q_{r}$ is positive the polarization is radial, and tangential in the case in which $Q_{r}$ is negative. On the other hand, $U_{r}$ represents the polarization rotated $45^{\circ}$ with respect to $Q_{r}$, as in the standard Stokes parameters. If the peaks are not oriented, then a polarization field with rotational symmetry is expected, and therefore, $Q_{r}$ and $U_{r}$ will not depend on $\phi$. This is not the case when the polarization field is described using the Cartesian Stokes parameters (examples of the expected patterns in this particular case can be seen in [13] and [7]). The azimuthal dependence introduced in this way is due to the inappropriate choice of the coordinate system, and it does not reflect the rotational symmetry of the polarization field.

The expected value of the multipolar profiles is calculated from eqs. (3.3) and eq. (5.3), taking into account that $\left\langle\hat{a}_{\ell m}\right\rangle=0$. We also assume that the first derivatives are zero $(\langle\eta\rangle=0)$. Therefore, the multipolar profiles $\left\langle T_{m}(\theta)\right\rangle$ only depend on the average of the peak height, mean curvature and eccentricity. Depending on how these mean values are constrained, different shapes are obtained. In order to have a peak, the condition imposed on the expected values of $\kappa$ and $\epsilon$ is the extremum constraint $(|\epsilon| \leq \sqrt{a}|\kappa|)$, which guarantees to have a maximum or minimum. In general, there is more freedom in choosing the value of $\nu$. For instance, if we are interested in peaks above a given threshold $\nu_{t}$, then its expected value must be calculated with the condition that $\nu>\nu_{t}$. Another possibility is to fix $\nu$ to a given value and study the pattern induced by the peak with that particular height. Since in this paper we are interested in the qualitative behaviour of peaks, this latter approach is used in the calculations.

Additionally, the polarization field of the peak in terms of $Q_{r}$ and $U_{r}$ is calculated from the $E$ and $B$ modes. Notice that, if the peak is located at the north pole, then $Q_{r}$ and $U_{r}$ coincide with the standard Stokes parameters on the sphere. Therefore, they can be calculated using its expansion in terms of the spin-weighted spherical harmonics.

Firstly, we consider peaks with rotational symmetry. In this case, the only expected contribution comes from the $m=0$ profile in the multipolar expansion (eq. (5.1)). The monopolar profiles are therefore given by

$$
\begin{gathered}
\left\langle T_{0}(\theta)\right\rangle=\sum_{\ell=0}^{\infty} \frac{2 \ell+1}{4 \pi}\left[b_{\nu}+b_{\kappa} \ell(\ell+1)\right] C_{\ell}^{T T} P_{\ell}(\cos \theta), \\
\left\langle Q_{r 0}(\theta)\right\rangle=-\sum_{\ell=2}^{\infty} \frac{2 \ell+1}{4 \pi} \sqrt{\frac{(\ell-2) !}{(\ell+2) !}}\left[b_{\nu}+b_{\kappa} \ell(\ell+1)\right] C_{\ell}^{T E} P_{\ell}^{2}(\cos \theta), \\
\left\langle U_{r 0}(\theta)\right\rangle=-\sum_{\ell=2}^{\infty} \frac{2 \ell+1}{4 \pi} \sqrt{\frac{(\ell-2) !}{(\ell+2) !}}\left[b_{\nu}+b_{\kappa} \ell(\ell+1)\right] C_{\ell}^{T B} P_{\ell}^{2}(\cos \theta),
\end{gathered}
$$

These profiles depend on the bias parameters $b_{\nu}$ and $b_{\kappa}$, which can be calculated from the expected value of $\nu$ and $\kappa$ :

$$
\left(\begin{array}{l}
b_{\nu} \sigma_{\nu} \\
b_{\kappa} \sigma_{\kappa}
\end{array}\right)=\Sigma^{-1}\left(\begin{array}{c}
\langle\nu\rangle \\
\langle\kappa\rangle
\end{array}\right)
$$


The matrix $\Sigma$ relating these quantities is the covariance matrix of $\nu$ and $\kappa$, described in Section 3 (see also eq. (B.6)). These profiles obtained for spherical symmetric peaks represent the generalization of the expressions in [13] for large angular distances (see appendix C). As it is expected, the temperature profile depends on the angular power spectrum $C_{\ell}^{T T}$. On the other hand, the coefficients in the multipolar expansion of the Stokes parameters are given by the cross-correlation between the temperature and the polarization fields. In the case of a spherically symmetric peak, it is possible to see that $Q_{r 0}$ describes the gradient of the polarization field, while $U_{r 0}$ represents the curl contribution. For this reason, $Q_{r 0}$ depends exclusively on the correlation of the temperature with $E$, which is the gradient of the polarization field, and $U_{r 0}$ depends on the correlation of $T$ with $B$, which is the curl contribution. If it is assumed that there is not any physical effect capable of rotating the polarization angle (e.g., birefringence [15]) or violating parity conservation [14] then the correlation between $T$ and $B$ vanishes, and hence the expected value of $U_{r 0}$ is zero.

The monopolar temperature profiles for maxima and different values of the peak height are represented in figure 2, showing the two effects due to the peak height and curvature biases. The curvature term contributes to the peak only at small scales, just modifying the peakedness of the profile. For large values of $\nu$, the monopolar peak profile tends to be proportional to the temperature correlation function, since the curvature bias becomes negligible (see figure 9 and the discussion in section 5.3 about the behaviour of the bias parameters). In the case of polarization, we consider only $Q_{r 0}$ because the $T B$ power spectrum is zero in the standard model. The monopolar profiles of $Q_{r}$ for maxima, conditioned to the value of $\nu$, are represented in figure 3. As in the temperature case, the contribution to the $Q_{r 0}$ profile for high $\nu$ comes from the correlation between the temperature and $Q_{r}$. The effect due to the curvature bias, present in profiles with small $\nu$, tends to modify the peaks of the $Q_{r 0}$ profile. The profiles represented in figures 2 and 3 are calculated for maxima, but equivalent results are obtained for minima.

In the case that $\langle\epsilon\rangle \neq 0$ (e.g., when the peaks are oriented towards some direction), then there is also a contribution to the quadrupolar profile $(m=2)$ in eq. (5.1):

$$
\begin{gathered}
\left\langle T_{2}(\theta)\right\rangle=b_{\epsilon} \sum_{\ell=0}^{\infty} \frac{2 \ell+1}{4 \pi} C_{\ell}^{T T} P_{\ell}^{2}(\cos \theta), \\
\left\langle Q_{r 2}(\theta)\right\rangle=-2 b_{\epsilon} \sum_{\ell=0}^{\infty} \frac{2 \ell+1}{4 \pi} \sqrt{\frac{(\ell-2) !}{(\ell+2) !}}\left[C_{\ell}^{T E} P_{\ell}^{+}(\cos \theta)+i C_{\ell}^{T B} P_{\ell}^{-}(\cos \theta)\right], \\
\left\langle U_{r 2}(\theta)\right\rangle=2 i b_{\epsilon} \sum_{\ell=0}^{\infty} \frac{2 \ell+1}{4 \pi} \sqrt{\frac{(\ell-2) !}{(\ell+2) !}}\left[C_{\ell}^{T E} P_{\ell}^{-}(\cos \theta)+i C_{\ell}^{T B} P_{\ell}^{+}(\cos \theta)\right] .
\end{gathered}
$$

In this case, the bias of the quadrupolar profiles is defined as $b_{\epsilon}=\langle\epsilon\rangle / \sigma_{\epsilon}$, which is proportional to the expected value of the eccentricity. The $\theta$ dependence in eqs. (5.6b) and (5.6c) is described by the functions:

$$
\begin{gathered}
P_{\ell}^{+}(x)=-\left[\frac{\ell-4}{1-x^{2}}+\frac{1}{2} \ell(\ell-1)\right] P_{\ell}^{2}(x)+(\ell+2) \frac{x}{1-x^{2}} P_{\ell-1}^{2}(x), \\
P_{\ell}^{-}(x)=-2\left[(\ell-1) \frac{x}{1-x^{2}} P_{\ell}^{2}(x)-(\ell+2) \frac{1}{1-x^{2}} P_{\ell-1}^{2}(x)\right] .
\end{gathered}
$$



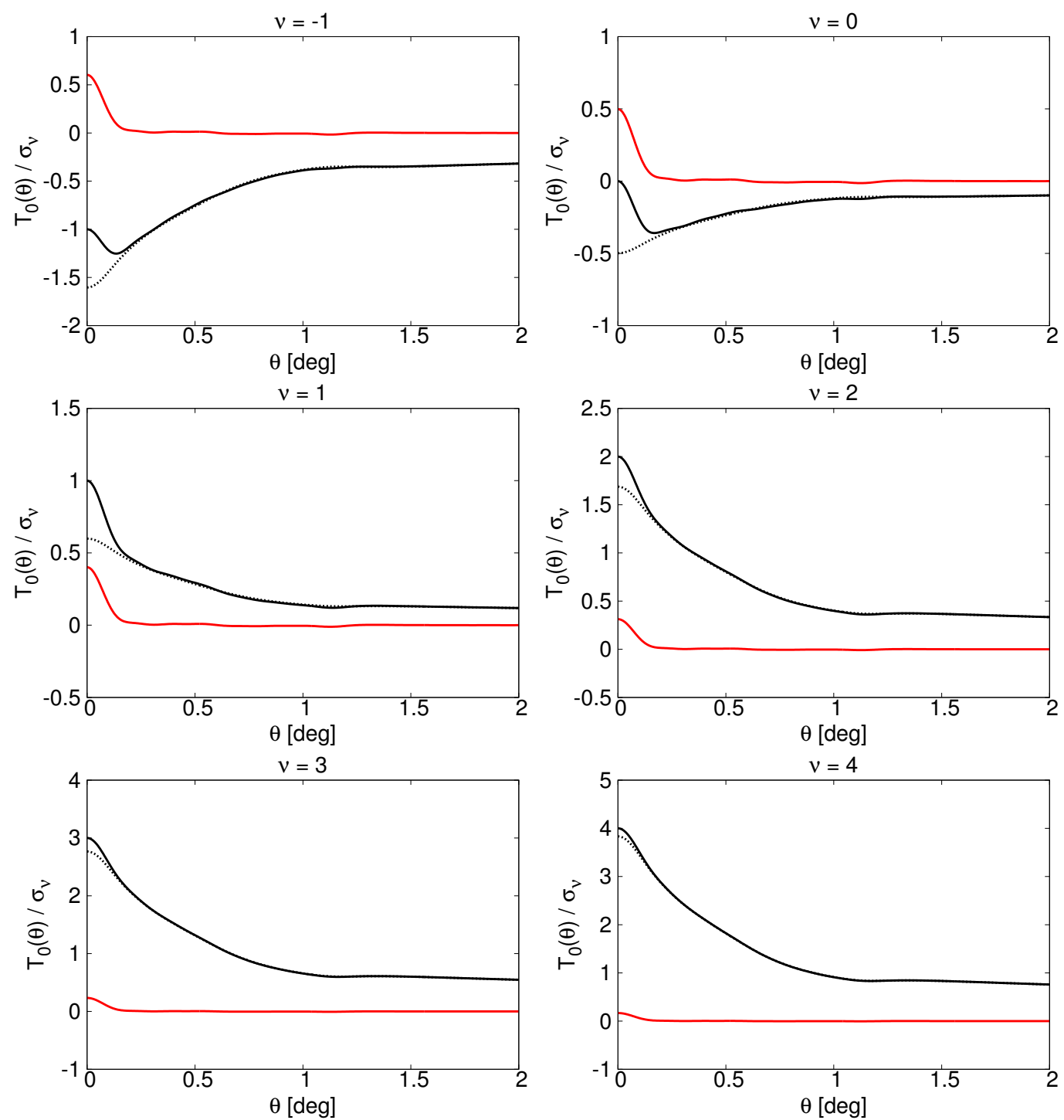

Figure 2. Spherically symmetric temperature profiles $T_{0}(\theta)$ for maxima conditioned to different peak heights. The dotted black lines depicts the contribution proportional to the temperature correlation function (peak height bias $b_{\nu}$ ), while the red line corresponds to the modification due to its Laplacian (mean curvature bias $b_{\kappa}$ ). The black solid line represents the total profile.

These functions arise in the analysis of any 2-spin field on the sphere (e.g., CMB polarization or weak lensing). They define the $\theta$ dependence of the 2 -spin spherical harmonics with $m=2$ as a function of $\ell[16,17]$. These expressions are undetermined in $\theta=0(x=1)$, but they have a continuous limit if the following values are adopted (see [17]):

$$
P_{\ell}^{ \pm}(1)= \pm \frac{1}{4} \frac{(\ell+2) !}{(\ell-2) !}
$$

The quadrupolar profiles in eqs. (5.6) are complex quantities whose phase represents a rotation of the system of reference. The principal axes coincide with the $x y$ axes when the eccentricity bias $b_{\epsilon}$ is real. Regarding the CMB polarization, one difference with respect to the 

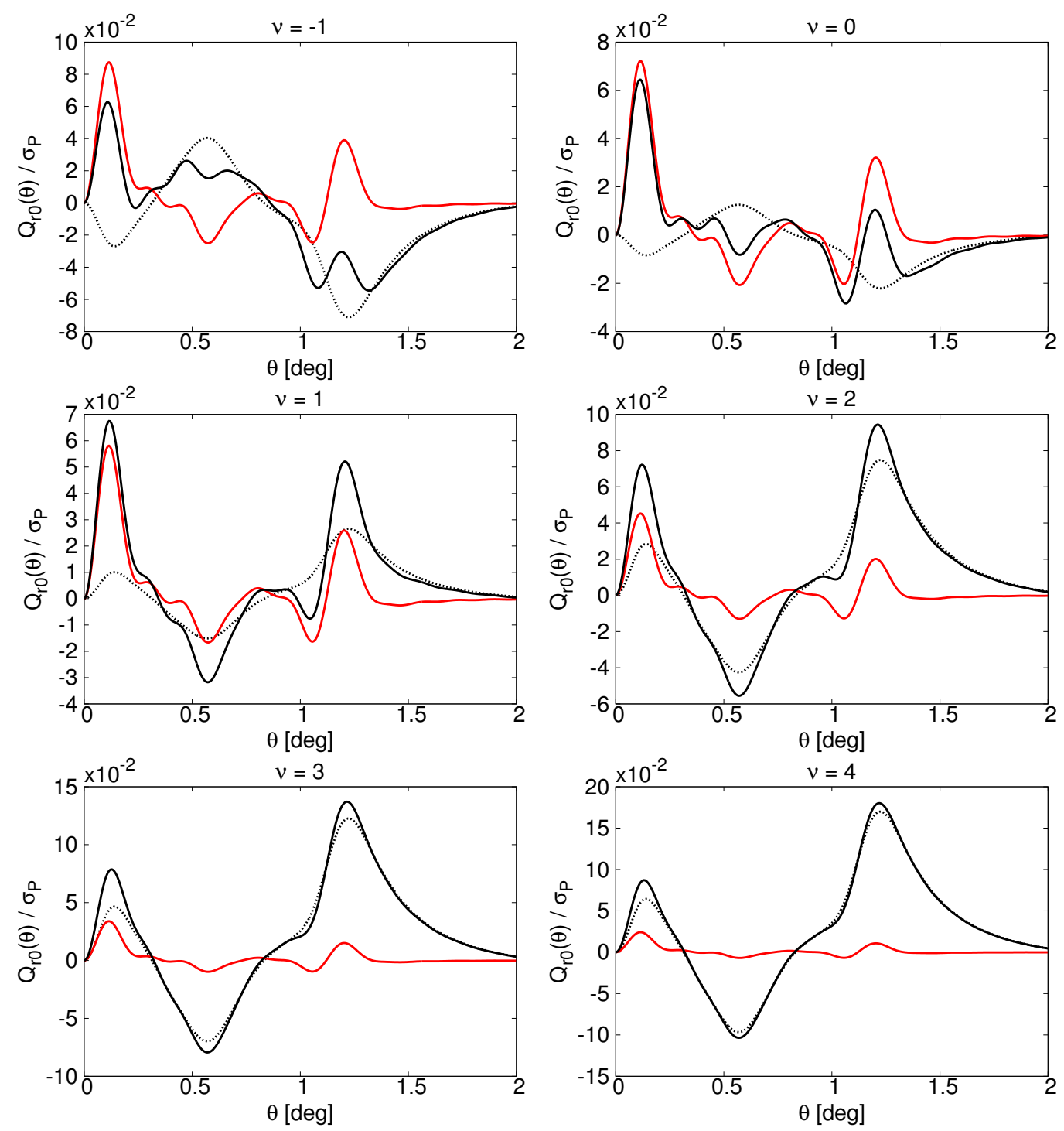

Figure 3. Monopolar profiles $Q_{r 0}(\theta)$ for different peak heights. The dotted black lines show the contribution due to the peak height bias $\left(b_{\nu}\right)$, while the red line is the modification caused by the mean curvature bias $\left(b_{\kappa}\right)$. The black solid line corresponds to the total profile. All these profiles are normalized by $\sigma_{P}=\sqrt{\left\langle Q^{2}\right\rangle+\left\langle U^{2}\right\rangle}$.

spherically symmetric case is that the polarization fields $E$ and $B$ contribute to both Stokes parameters $Q_{r}$ and $U_{r}$. The Stokes parameters in polar coordinates describe properly peaks with rotational symmetry. However, when the peak has nonzero eccentricity, the gradient and curl contributions are mixed due to the elongation of the peak. The effect of the eccentricity bias on the temperature and polarization peak shapes is represented in figures 4-6 as a function of the peak height. In these figures, the mean value of the eccentricity has been calculated from the probability density distribution in eq. (4.1), imposing the condition that $\epsilon$ is real $(\alpha=0)$. Geometrically, this is equivalent to orient the peak, such that the principal axes coincide with the Cartesian system of reference. Additionally, the 2-dimensional shape of 
temperature peaks and its effect on the Stokes parameters are shown in figures 7 and 8 .

Let us remark that the multipolar profiles have already been used to test the standard cosmological model with the Planck temperature and polarization data [7]. ${ }^{2}$ The orientation of peaks in that work is performed by selecting the principal axes in the inverse Laplacian of the temperature. This allows to reduce the noise contribution in order to have a better estimation of the orientation axes. On the other hand, the theoretical calculations in the present paper are done directly in the temperature field, but the formalism can be trivially generalized so that the peak is selected and oriented in any derived field. For instance, it is possible to select the peak in a smoothed version of the temperature (in particular the inverse Laplacian), in order to reduce the noise or study the physics of peaks at different scales. In this sense, the work in this paper complements the study in [7] giving a theoretical background, which is completely general and can be applied to many situations.

\subsection{Profiles in real space}

In this subsection, we provide an alternative description of the peak profiles, in which they are expressed in terms of derivatives of different correlation functions, depending on which field is considered and where the peak is selected. In the following, it is assumed that the peak is located in the temperature field and its effect on a general field $X$, which can be $T, E$, $B, Q_{r}$ or $U_{r}$, is studied. It is straightforward to generalize this formalism for peaks selected in any other field replacing $T$ by that field. Using vector notation we have that $\left\langle X_{m}(\theta)\right\rangle$ can be written as the following dot products:

$$
\begin{aligned}
& \left\langle X_{0}(\theta)\right\rangle=\mathbf{b}_{0}^{\dagger} \mathbf{C}_{0}^{T X}(\theta), \\
& \left\langle X_{2}(\theta)\right\rangle=\mathbf{b}_{2} \mathbf{C}_{2}^{T X}(\theta),
\end{aligned}
$$

where the biases $\mathbf{b}_{0}$ and $\mathbf{b}_{2}$ are defined as

$$
\mathbf{b}_{0} \equiv\left(\begin{array}{c}
b_{\nu} \\
b_{\kappa}
\end{array}\right), \quad \mathbf{b}_{2} \equiv b_{\epsilon}
$$

The biases concerning the scalar degrees of freedom $\nu$ and $\kappa$ are combined in the vector $\mathbf{b}_{0}$, while the bias related to the eccentricity is denoted by $\mathbf{b}_{2}$ for convenience. The $\theta$-dependence of the multipolar profiles is calculated from the correlation function $C^{T X}(\theta)$ :

$$
\mathbf{C}_{0}^{T X}(\theta)=\left(\begin{array}{c}
1 \\
-\nabla^{2}
\end{array}\right) C^{T X}(\theta), \quad \mathbf{C}_{2}^{T X}(\theta)=\left(\not \partial^{*}\right)^{2} C^{T X}(\theta)
$$

The first component of the vector $\mathbf{C}_{0}^{T X}$ is the correlation function itself, while the second one is minus its Laplacian. On the other hand, the function $\mathbf{C}_{2}^{T X}$ defining the quadrupolar profile is written as a second order covariant derivative of the correlation function.

\footnotetext{
${ }^{2}$ In [7], the multipolar profiles of polarization are defined expanding the quantity $Q+i U$, where the Stokes parameters are given in Cartesian coordinates (only valid in the flat approximation). The profiles $P_{m}$ arising in this expansion are related to the ones used in this work as follows:

$$
\begin{gathered}
P_{0}=Q_{r 2}-i U_{r 2}, \\
P_{2}=Q_{r 0}, \\
P_{4}=Q_{r 2}+i U_{r 2} .
\end{gathered}
$$
}



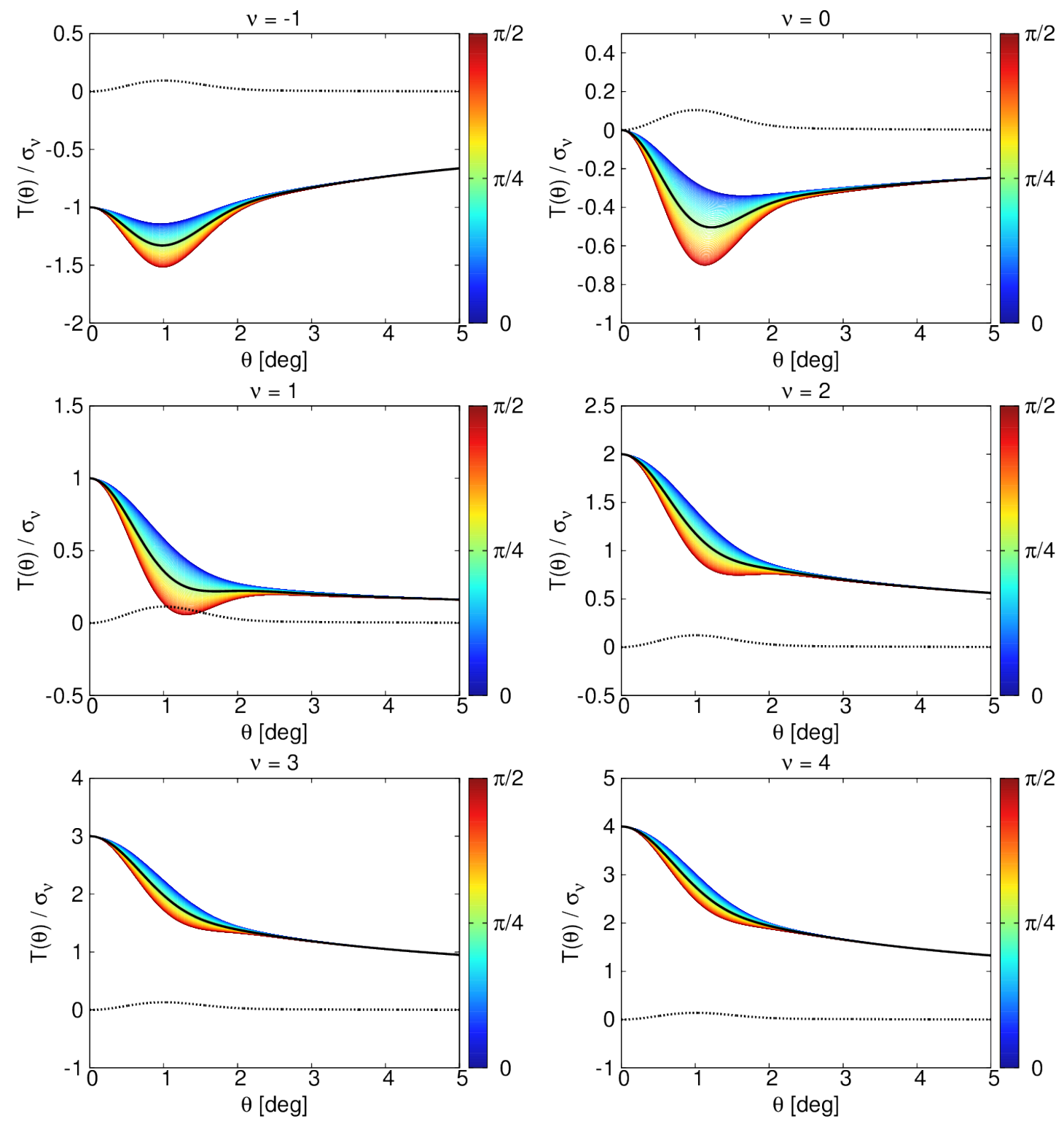

Figure 4. The effect of the eccentricity on the temperature profile for different $\nu$. The principal axes of the peak are oriented according to the Cartesian system of reference, which implies that $\epsilon$ is real and the eccentricity bias is given by $b_{\epsilon}=\langle|\epsilon|\rangle / \sigma_{\epsilon}$. The black solid line depicts the spherically symmetric profile $(m=0)$. The color scale represents how the peak profile varies as a function of the azimuthal angle $\phi$. The maximum and minimum elongations are reached at $\phi=0$ and $\phi=\pi / 2$ respectively. The quadrupolar profiles $(m=2)$ are represented by black dotted lines. In this figure, the temperature field is filtered by a Gaussian of FWHM $1^{\circ}$.

The quantities defined in eq. (5.11), which determine the shape of the peak, are different derivatives of the correlation function. Indeed, these derivatives are the cross-correlations of the field $X$ with the peak degrees of freedom. For instance, the Laplacian of the correlation function $\nabla^{2} C^{T X}(\theta)$ is proportional to the correlation of the mean curvature $\kappa$ and the field $X$, that is, $\langle\kappa X\rangle$. On the other hand, the derivative $\left(\not^{*}\right)^{2} C^{T X}(\theta)$ is proportional to the 

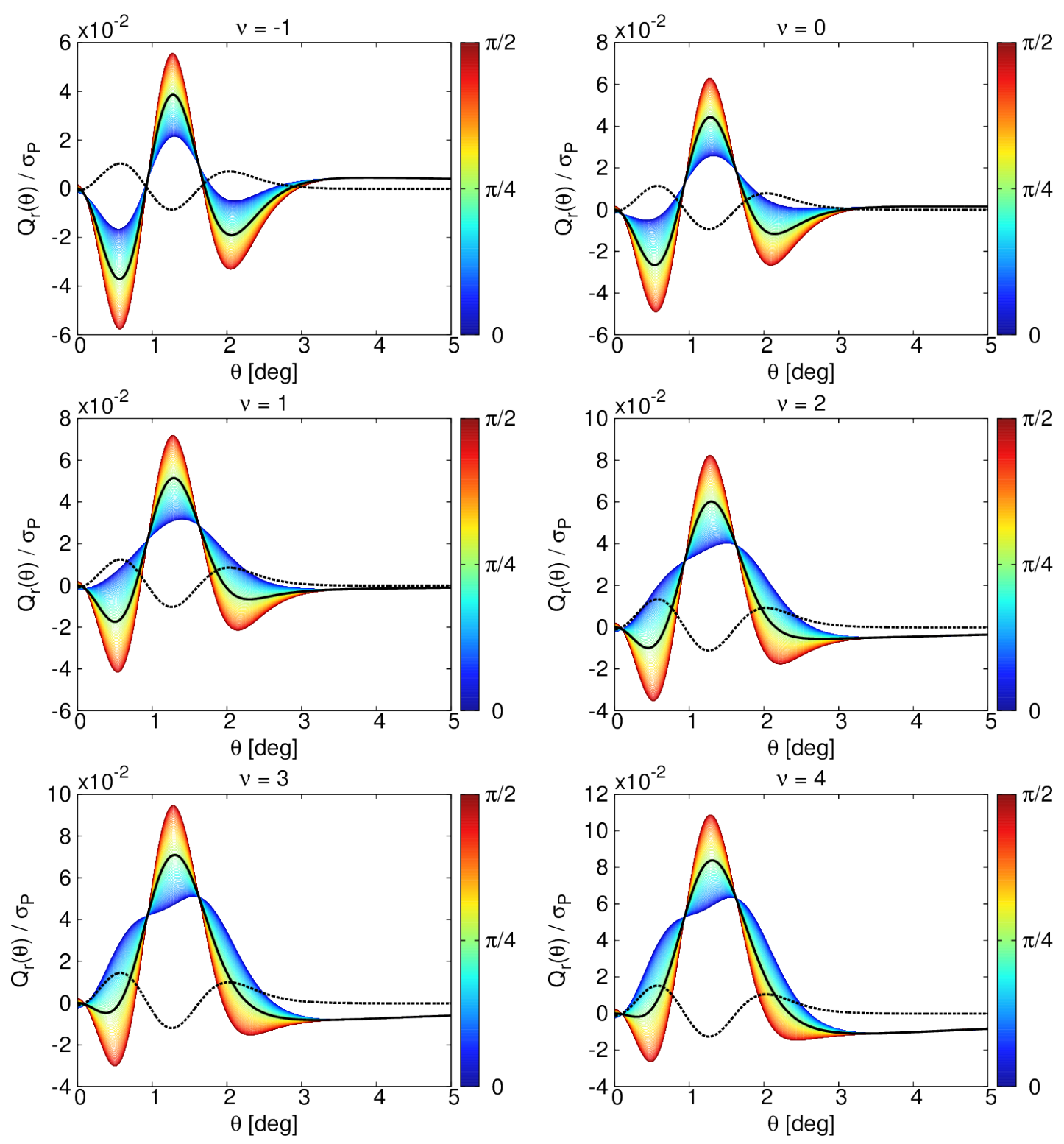

Figure 5. The effect of the eccentricity on the $Q_{r}$ profile for different $\nu$. The peaks are oriented in the same way than in figure 4 . The black solid line corresponds to the spherically symmetric profile $(m=0)$. The color scale represents how the profile varies as a function of the azimuthal angle $\phi$. The maximum and minimum elongations are reached at $\phi=0$ and $\phi=\pi / 2$ respectively. The quadrupolar profiles $Q_{r 2}(\theta)$ are represented by black dotted lines. In this figure, the peak is selected in the temperature field, filtered by a Gaussian of FWHM $1^{\circ}$. All these profiles are normalized by $\sigma_{P}=\sqrt{\left\langle Q^{2}\right\rangle+\left\langle U^{2}\right\rangle}$.

correlations $\langle\epsilon X\rangle$, while the correlation function itself is proportional to $\langle\nu X\rangle$. The fact that the field $X$ is correlated with the peak degrees of freedom is the reason why any constraint on the peak variables $\nu, \kappa$ and $\epsilon$ modifies the shape of the peak. 


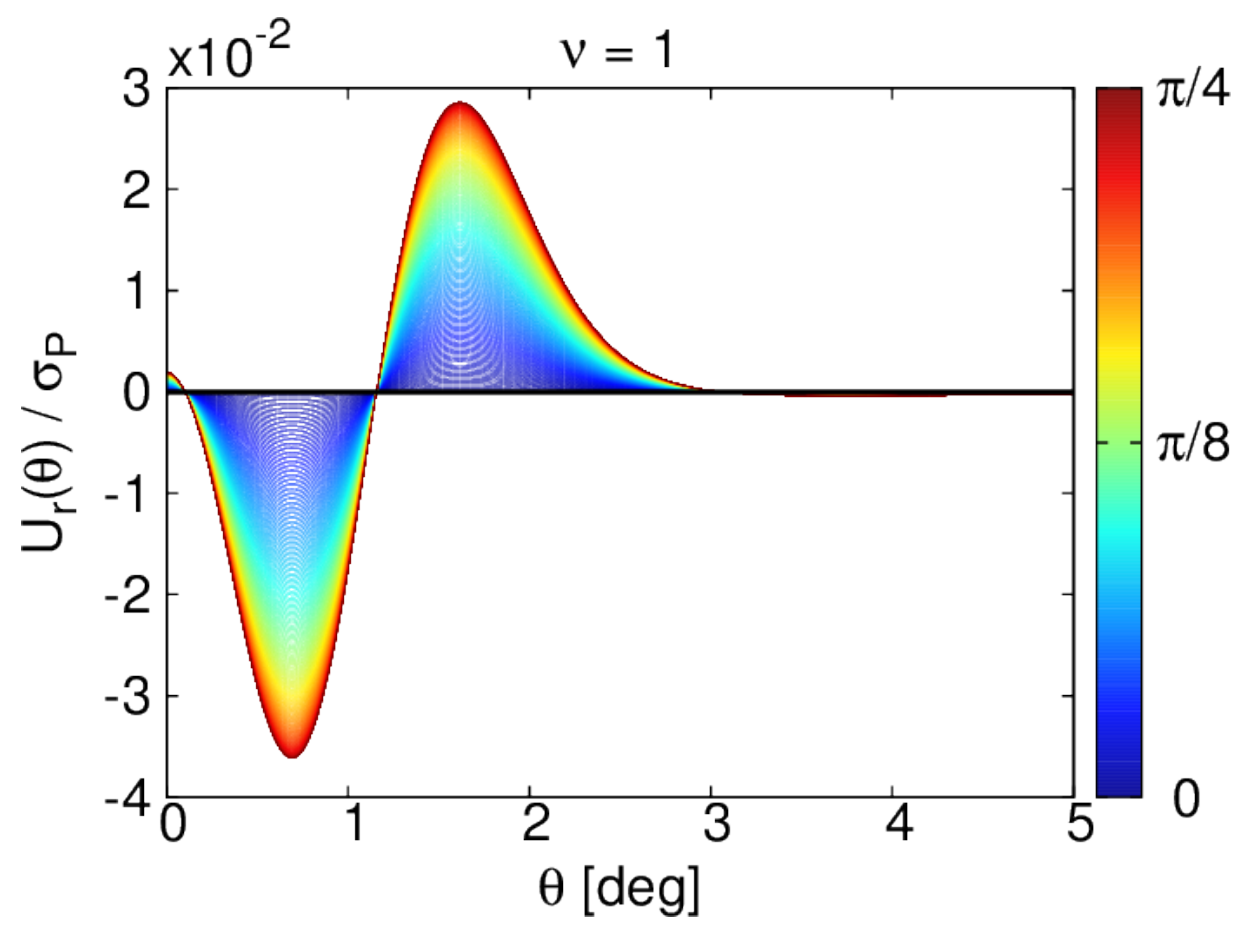

Figure 6. The effect of the eccentricity on the $U_{r}$ profile for $\nu=1$. The peaks are oriented in the same way than in figure 4 . The color scale represents how the profile varies as a function of the azimuthal angle $\phi$. This profile vanishes for $\phi=0$ and it increases until reaching the maximum contribution at $\phi=\pi / 4$. Different values of $\nu$ only change the amplitude of this profile following the dependence of $\left|b_{\epsilon}\right|$ as a function of the peak height (see figure 9). In this figure, the peak is selected in the temperature field, filtered by a Gaussian of FWHM $1^{\circ}$. The profile is represented normalizing by $\sigma_{P}=\sqrt{\left\langle Q^{2}\right\rangle+\left\langle U^{2}\right\rangle}$.
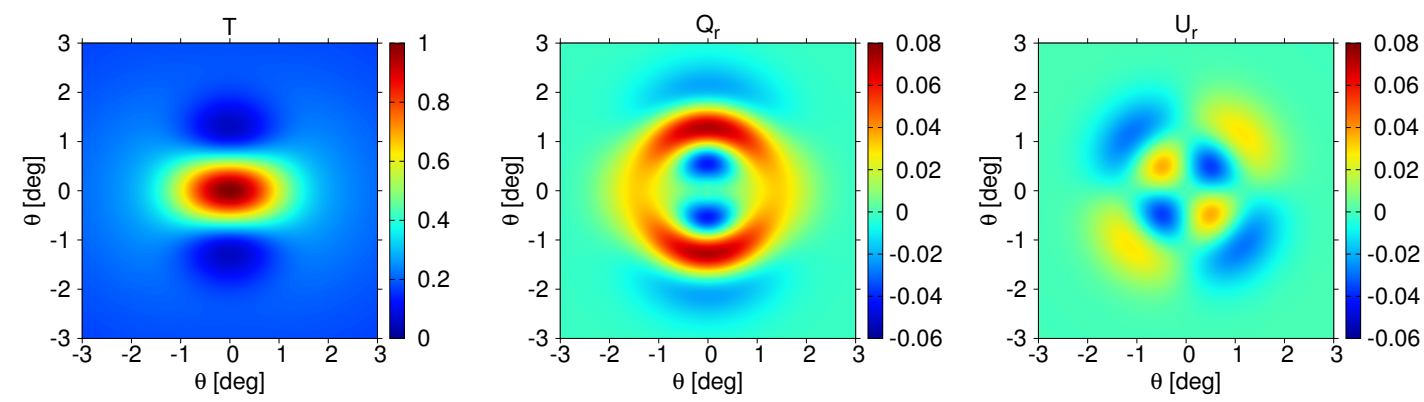

Figure 7. The 2-dimensional shape of peaks with eccentricity for oriented peaks with $\nu=1$. The panels from left to right represents $T, Q_{r}$ and $U_{r}$. In this figure, only the temperature field is filtered with a Gaussian of FWHM $1^{\circ}$. The units of color scales are given in terms of $\sigma_{\nu}$ for the temperature, and $\sigma_{P}$ for the Stokes parameters.

\subsection{Bias discussion}

The terms contributing to the multipolar profiles in eqs. (5.9) arise from different peak selection biases. There are three conditions that can be imposed on peaks: constraints on the 

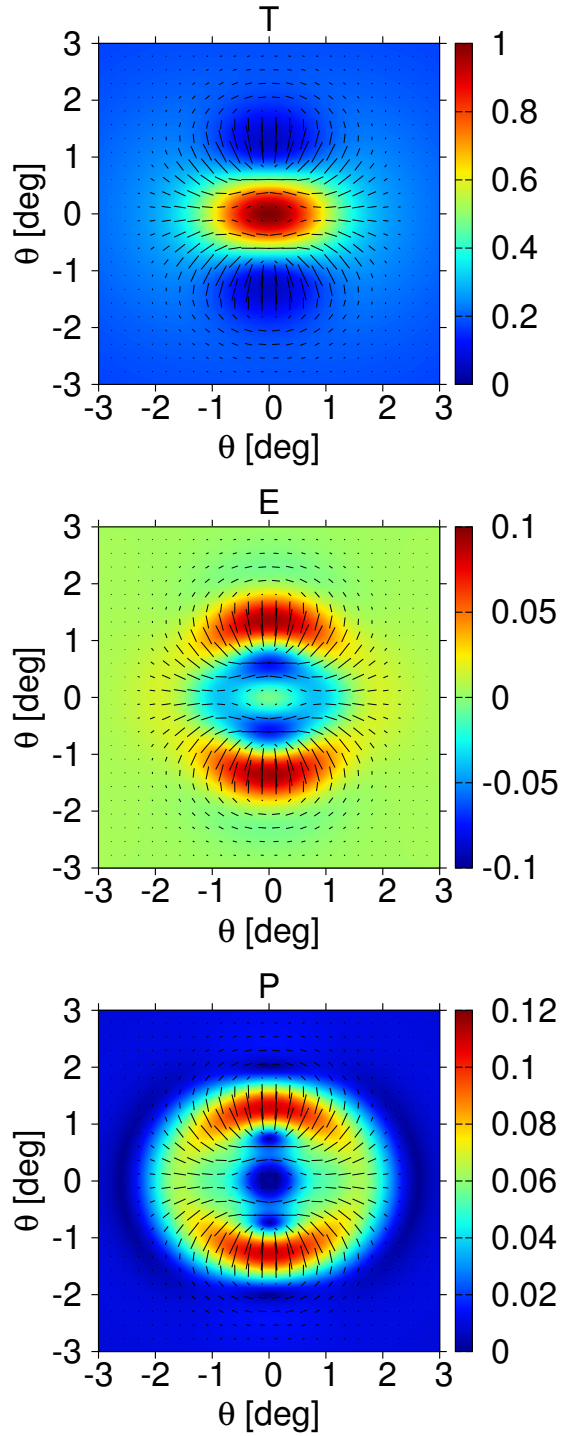
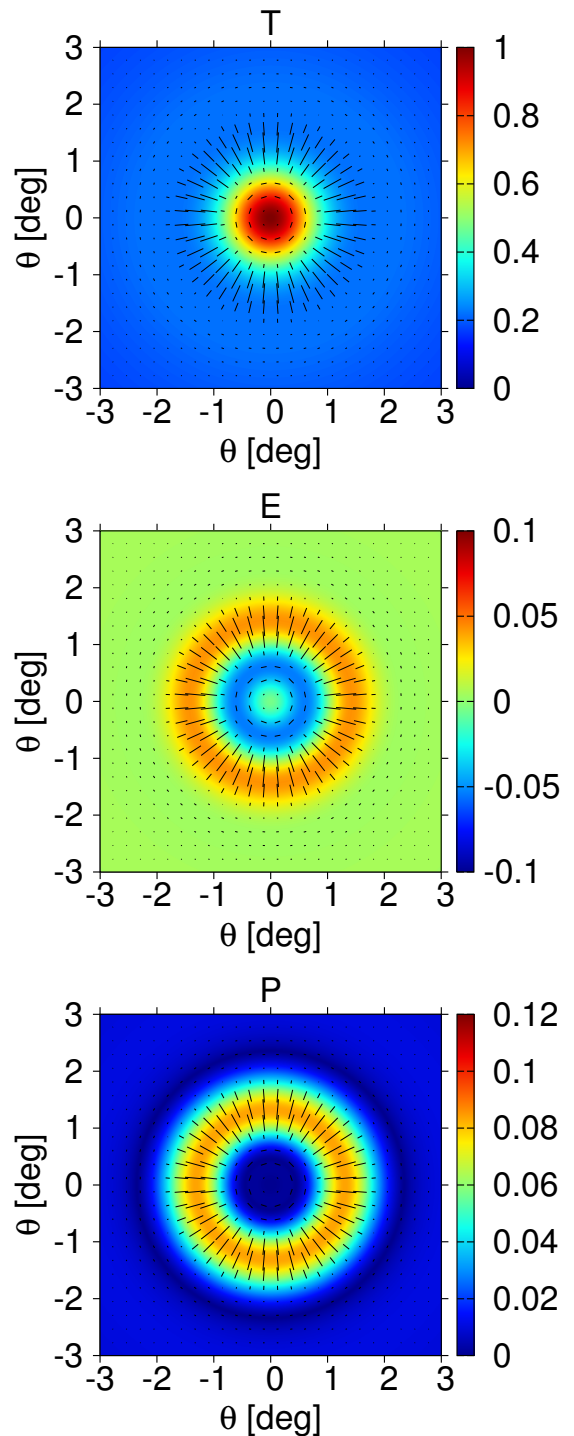

Figure 8. The 2-dimensional shape of peaks for $\nu=1$. In the left panels, it is considered a oriented peak with eccentricity, whilst in the right panels it is represented a spherical symmetric peak. In all these figures, the polarization directions are drawn over it. The length of the headless vectors is proportional to the polarization degree. Upper row: the color map represents the temperature pattern induced by the peak. Middle row: in this case the color map depicts the $E$-mode polarization. Lower row: it is represented $P \equiv \sqrt{Q^{2}+U^{2}}$, which describes the degree of polarization. In this figure, only the temperature field is filtered with a Gaussian of FWHM $1^{\circ}$. The units of color scales are given in terms of $\sigma_{\nu}$ for the temperature, and $\sigma_{P}$ for the $E$-mode and $P$.

peak height, the condition of being a maximum or minimum and constraints on the orientation of the peak. The condition of being an extremum affects to the mean curvature and the eccentricity (see section 4). Hereafter, the bias parameters are calculated conditioning to the value of $\nu$.

The biases for maxima are represented in figure 9 as a function of the peak height. In the high- peak limit, the maximum selection has no effect on the profile because it is more 

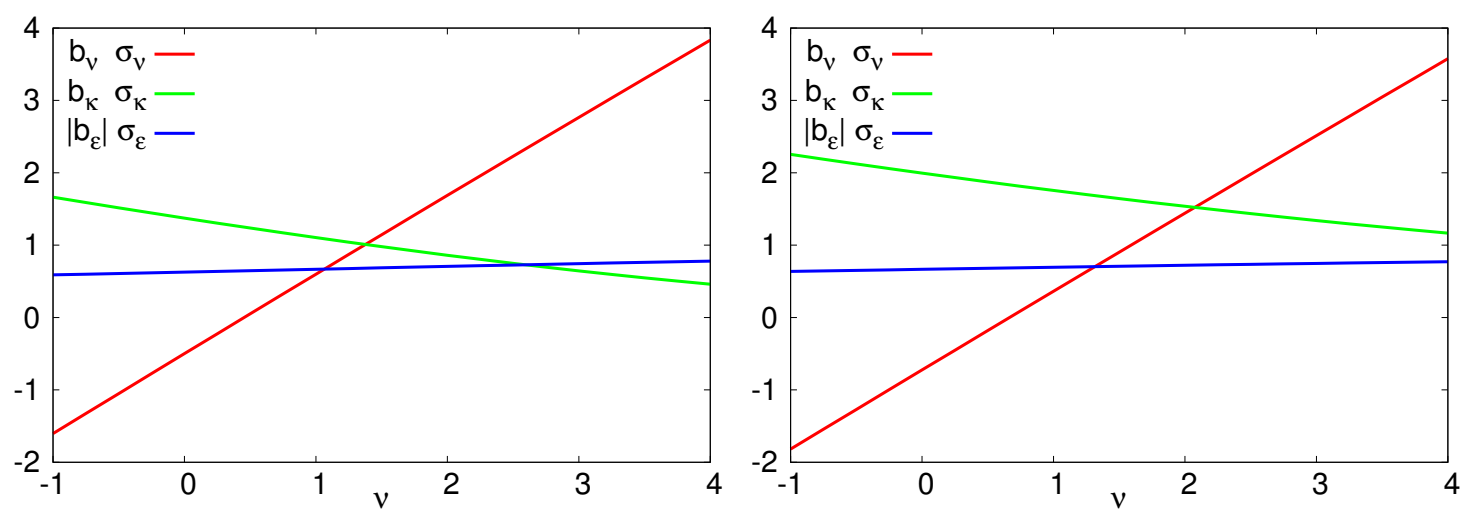

Figure 9. Biases of the peak profile for maxima as a function of the peak height $\nu$. The curvature and the eccentricity are marginalised, while the peak height is conditioned to a given value. Left: The one-point probability is used for averaging the peak variables and calculating the biases. Right: The biases are calculated using the peak number density.

likely that a peak with high $\nu$ is a maximum, without any additional bias on the curvature. Therefore, the curvature bias $b_{\kappa}$ approaches to zero for high $\nu$ (the expected value of the mean curvature is $\langle\kappa\rangle \sim \rho \nu$ for large values of $\nu$ ). We arrive at the same conclusion if we consider minima with extreme negative values of $\nu$. On the other hand, the peak height bias $b_{\nu}$ approaches to $\nu / \sigma_{\nu}$ in the high-peak limit (see figure 9). Hence, the radial profile of high peaks is proportional to the correlation function.

Finally, we consider constraints on the eccentricity. If the peaks are oriented according to its principal axes, then the mean value $\langle\epsilon\rangle$ is not zero, introducing a bias in the value of $\epsilon$. The quadrupolar profile $(m=2)$ in eq. $(5.9 \mathrm{~b})$, which breaks the rotational symmetry and introduces an azimuthal dependence in the peak shape, is proportional to the bias $b_{\epsilon}=\langle\epsilon\rangle / \sigma_{\epsilon}$. As this bias is a complex number whose argument only has information about the orientation angle, then the statistical properties of the eccentricity are only in its modulus $\left|b_{\epsilon}\right|$. In the high-peak limit, the modulus of the eccentricity bias approaches to $\left|b_{\epsilon}\right|=\sqrt{\pi} / 2 \sigma_{\epsilon}$.

In figure 9 , we consider two different ways of calculating the biases. In one case, the one-point probability (eq. (4.1)) is used for averaging the peak variables and, in the other case, it is used the number density of peaks (eq. (4.2)). Each of these approaches are useful in different situations (see Section 4 for a discussion). Although the biases must be independent of the probability used for their calculation in the high-peak limit, differences can be seen for large values of $\nu$.

The eccentricity is a complex number whose phase describes the orientation of the peak. It is possible to remove this phase choosing the principal axes of the peak as the reference system. In this particular case, both the eccentricity $\epsilon$ and the bias $b_{\epsilon}$ are real. Combining eqs. (5.9), it can be shown that the expected value $\langle T(\theta, \phi)\rangle$ is a biased correlation function, where the bias can be seen as the operator

$$
b=b_{\nu}-\left(b_{\kappa}-2\left|b_{\epsilon}\right|\right) \partial_{x}^{2}-\left(b_{\kappa}+2\left|b_{\epsilon}\right|\right) \partial_{y}^{2},
$$

where we consider the principal axes as the $x y$-coordinates. This bias is non-local because it contains partial derivatives. Furthermore, it is non-isotropic due to the fact that the derivatives along the principal directions have different bias. Only when there is no eccentricity 
bias $\left(b_{\epsilon}=0\right)$, we recover isotropy. The bias in eq. (5.12) is a generalization of the one in [10] for peaks with eccentricity.

\section{Covariance of the multipolar profiles}

In this section, the covariance of the multipolar profiles are calculated. As in the previous section, both the temperature and the Stokes parameters are expanded in terms of the multipolar profiles. In the case of peaks with spherical symmetry, only the scalar profile $(m=0)$ contributes to the peak local shape. Conversely, if peaks are selected with eccentricity, then the quadrupolar profile $(m=2)$ is also non-zero. In this case, obviously, the multipolar profiles with $m=0$ and $m=2$ have all the information concerning the peak shape.

In general, the covariance between the multipolar profiles of the field $X$ and $Y$ can be written as the sum of two contributions:

$$
\left\langle X_{m^{\prime}}^{*} Y_{m}\right\rangle=\left\langle X_{m^{\prime}}^{*} Y_{m}\right\rangle_{\text {intr. }}+\left\langle X_{m^{\prime}}^{*} Y_{m}\right\rangle_{\text {peak }}
$$

where the intrinsic covariance $\left\langle X_{m^{\prime}}^{*} Y_{m}\right\rangle_{\text {intr. }}$ represents the correlations of the multipolar profiles, independently whether a peak is selected or not. The second part $\left\langle X_{m^{\prime}}^{*} Y_{m}\right\rangle_{\text {peak }}$ is a modification of the intrinsic covariance due to the fact that a peak is present in the field. In general, the contribution of the peak is a suppression of the intrinsic covariance caused by the reduction of the field randomness when the peak variables are constrained.

The intrinsic covariance is given by

$$
\left\langle X_{m^{\prime}}^{*}\left(\theta^{\prime}\right) Y_{m}(\theta)\right\rangle_{\text {intr. }}=\delta_{m m^{\prime}} \sum_{\ell=m}^{\infty} \frac{2 \ell+1}{4 \pi} \frac{(\ell-m) !}{(\ell+m) !} C_{\ell}^{X Y} P_{\ell}^{m}(\cos \theta) P_{\ell}^{m}\left(\cos \theta^{\prime}\right)
$$

Whilst this covariance is only determined by the angular cross-power spectrum of the fields and affects to the whole range of $m$-values, the peak covariance depends on how the peak variables are selected. In addition to the covariance of the $m=0$ and $m=2$ profiles, the peak also modifies the covariance of the multipolar profile with $m=1$, which is associated to the first derivative. The condition of having a critical point $(\eta=0)$ implies that the expected value of the dipolar profile is zero, and for this reason we have not considered the $m=1$ profile in the peak shape analysis (see Section 5). However, as the covariance of the field is affected by the constraint on the first derivative, the parameter $\eta$ must be included in the analysis of this section.

The contribution of the peak to the field covariance is caused by the particular constraints on the peak degrees of freedom (for instance, imposing the extremum constraint, the peak height above a given threshold, or the first derivative equal to zero). These constraints modify how the peak variables are distributed with respect to the case without peak selection. In the following, the covariance matrix of $\nu, \kappa, \eta$ and $\epsilon$, when peak variables are unconstrained, is denoted by $S$. Once the peak is selected, the change in this covariance is parametrized by the matrix $\Delta S$, which is defined as the difference between the covariance of $\nu, \kappa, \eta$ and $\epsilon$, with and without the peak constraints imposed. The bias of $S$ is defined as the matrix:

$$
B_{S}=B(\Delta S) B^{\dagger}
$$


where the four-dimensional matrix $B$ is given by the inverse of $S$, normalizing the rows by the corresponding variances of the peak variables. That is,

$$
B=\left(\begin{array}{cccc}
\sigma_{\nu}^{-1} & 0 & 0 & 0 \\
0 & \sigma_{\kappa}^{-1} & 0 & 0 \\
0 & 0 & \sigma_{\eta}^{-1} & 0 \\
0 & 0 & 0 & \sigma_{\epsilon}^{-1}
\end{array}\right) S^{-1}
$$

The bias of the covariance $B_{S}$ in eq. (6.3) is a linear transformation of the matrix $\Delta S$. Therefore, if the peak variables are not constrained (i.e., $\Delta S=0$ ), the bias $B_{S}$ is also zero. For convenience, the bias matrix $B_{S}$ is separated in different blocks taking into account the different spin of the peak variables:

$$
B_{S}=\left(\begin{array}{lll}
B_{00} & B_{01} & B_{02} \\
B_{10} & B_{11} & B_{12} \\
B_{20} & B_{21} & B_{22}
\end{array}\right) .
$$

This matrix is Hermitian by construction, and therefore $B_{i j}=B_{j i}^{*}$. The reason of this decomposition is that the peak variables affect to the different multipolar profiles depending on their spin. The two-dimensional matrix $B_{00}$ represents the bias of the covariance of the scalar degrees of freedom $\left(\nu\right.$ and $\kappa$ ), while $B_{11}$ and $B_{22}$ are the biases of the variances of the first derivative $(\eta)$ and the eccentricity $(\epsilon)$, respectively. Likewise, due to the peak selection process, it is possible to have correlations between different peak variables, which are described by the off-diagonal terms of $B_{S}$ (for instance, the extremum constraint $|\epsilon| \leq|\sqrt{a}| \kappa \mid$ introduces correlations between $\kappa$ and $\epsilon$ ). In the particular case of peaks where the first derivative is set to zero by definition, the bias in the covariance of $\eta$ is $B_{11}=-1 / \sigma_{\eta}^{2}$ and there is no correlation between $\eta$ and the rest of degrees of freedom, which leads to $B_{01}=B_{12}=0$. Finally, the peak covariance is calculated using the bias matrix $B_{S}$ :

$$
\left\langle X_{m^{\prime}}^{*}\left(\theta^{\prime}\right) Y_{m}(\theta)\right\rangle_{\text {peak }}=\mathbf{C}_{m^{\prime}}^{T X}\left(\theta^{\prime}\right)^{\dagger} B_{m^{\prime} m} \mathbf{C}_{m}^{T Y}(\theta),
$$

where $\mathbf{C}_{m}^{T X}$ for $m=0,2$ are defined in eq. (5.11). In the particular case of $m=1$, this quantity is given by the covariant derivative of the correlation function:

$$
\mathbf{C}_{1}^{T X}(\theta)=\not \partial^{*} C^{T X}(\theta) .
$$

In eq. (6.6), it is assumed that the peak is selected in temperature, but it can be generalized for peaks in any other field replacing $T$ by that field.

When a peak is present in the field, the covariance is reduced coherently depending on how the peak variables are constrained. For instance, if the peak height $\nu$ is fixed to a given value or selected above a threshold, the field at the centre is constrained, and therefore it is expected that the variance at $\theta=0$ is reduced. In figure 10, it is represented the covariance of the $m=0$ and $m=2$ profiles for peaks selected in temperature with $\nu>1$. It is possible to see that the effect of the peak on the covariance mainly affects the TT part, while the covariances concerning the Stokes parameters are dominated by the intrinsic term. This fact is produced because the peak covariance of the Stokes parameters is proportional to the square of the $T E$ correlation, which is subdominant with respect to the intrinsic fluctuations of the field. This is not the case for temperature, where the presence of a peak modifies drastically the covariance around the centre and introduces correlations between different $\theta$. Additionally, 

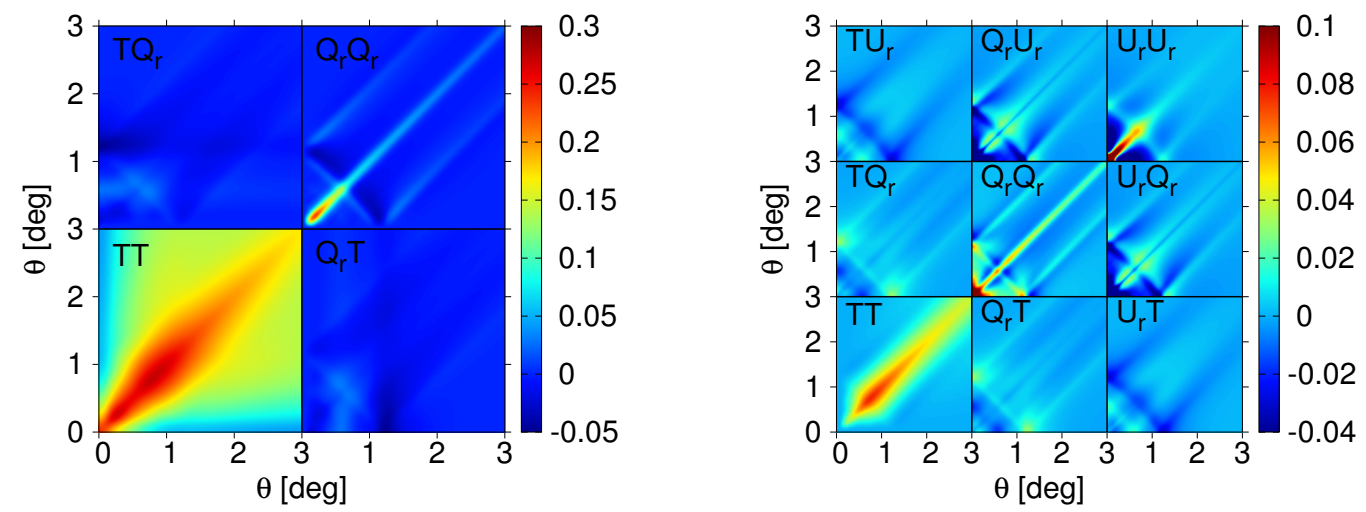

Figure 10. Covariance of the $m=0$ (left) and $m=2$ (right) profiles for peaks selected in temperature with $\nu>1$. Each field is normalized by the corresponding standard deviation $\left(\sigma_{\nu}\right.$ for the temperature and $\sigma_{P} / \sqrt{2}$ for each of the Stokes parameters).

it is possible to consider the covariance between the monopolar and the quadrupolar profiles. However, the intrinsic part vanishes and the effect of the peak is small in this case.

If we are interested in analysing the two-dimensional pattern instead of the individual multipolar profiles, it is necessary to calculate the covariance of the field $X(\theta, \phi)$. Since all the information of the field is contained in the multipolar profiles, the field covariance can be calculated from the covariance of the multipolar profiles:

$$
\left\langle X\left(\theta^{\prime}, \phi^{\prime}\right) X(\theta, \phi)\right\rangle=\sum_{m, m^{\prime}=-\infty}^{\infty}\left\langle X_{m^{\prime}}^{*}\left(\theta^{\prime}\right) X_{m}(\theta)\right\rangle e^{i\left(m \phi-m^{\prime} \phi^{\prime}\right)}
$$

This covariance can also be split into the intrinsic and the peak contributions. As it is expected, the intrinsic part obtained from eq. (6.2) leads to the field correlation function, depending on the separation of the two points. On the other hand, the peak contribution is modelled by the covariance of the multipolar profiles with $m=0,1,2$ in eq. (6.6). These terms introduce a inhomogeneous correlation function around the peak, which can be also anisotropic if the peak has eccentricity. In figure 11, it is represented the variance of each point around an oriented peak selected in temperature. In the region close to the centre, the variance is suppressed with respect to the intrinsic variance. The quadrupolar pattern present in this figure is a consequence of the peak eccentricity. Whilst the peak has a strong effect on the variance of the temperature field, the variances of the Stokes parameters are modified in less than $1 \%$.

\section{Physical interpretation of the peak patterns}

The azimuthally averaged temperature peak patterns, where the effect of the eccentricity has been averaged out at zero, are essentially given by the correlation function between $T$ and the field where the pattern is imprinted, which can be either $T$ or the polarization fields. Modifications due to the peak curvature can arise in the low-peak limit, but this effect is only manifested in the region close to the centre of the peak and it has not influence in the physical behaviour of the profiles at large scales. In the high-peak limit, or for distances greater than the correlation of $\nabla^{2} T$, the physics of peaks is the same as the one causing the shape of the 

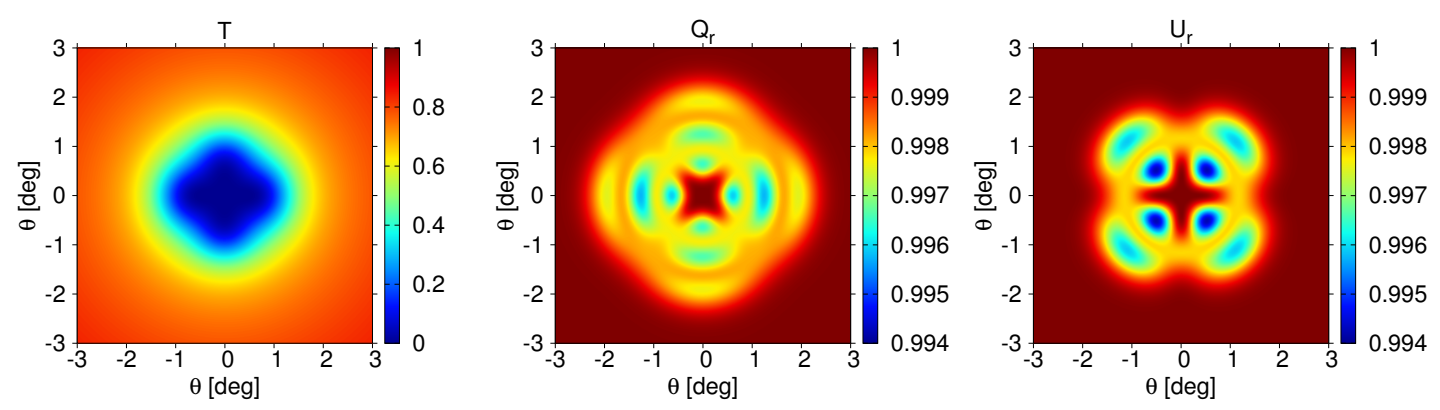

Figure 11. Variance of the temperature (left), $Q_{r}$ (middle) and $U_{r}$ (right) fields around a peak selected in $T$ smoothed by a Gaussian of FWHM $1^{\circ}$. The peak height is conditioned to be $\nu=1$, and therefore these variances correspond to the patterns in figure 7 . Each field is normalized by its variance corresponding to the case of no peak selection. It is possible to see that the peak barely alters the variance of the Stokes parameters $(<1 \%)$, whilst the temperature variance is drastically decreased in the region of the peak.

corresponding temperature cross-correlation functions. For instance, the ring structure seen in the $Q_{r}$ profile (figure 3) is an effect of the baryon acoustic oscillations produced at scales smaller than the sound horizon size at the decoupling epoch, which are also present in the $T Q_{r}$ (or $T E$ ) correlation [7, 13].

In this paper, we analyse the effect of the eccentricity in both temperature and polarization patterns. In the case of the temperature, the eccentricity of the peak affects to the second order derivatives at the centre adding a directional dependence. This effect modifies essentially the small scales since the eccentricity term is proportional to $\sim \ell^{2}$. However, the eccentricity is noticeable at scales up to the sound horizon size. The acoustic oscillations produced inside of a non-spherical potential propagate the anisotropy from the centre to the horizon size. In contrast, for scales greater than the horizon, the physics is dominated by gravity, which is not sensitive to the local geometry of the potential well, and the spherical symmetry is therefore recovered. In figure 4 , we can see this effect, where the peak profile is represented for different azimuthal angle. The quadrupolar profile $T_{2}(\theta)$ characterizes the effect of the eccentricity on the temperature peak as a function of $\theta$. The eccentricity does not alter the peak height at the centre, and therefore $T_{2}(\theta)$ vanishes at $\theta=0$. However, this term contributes at scales within the sound horizon. For larger scales, $T_{2}(\theta)$ goes to zero and the peak becomes spherically symmetric.

In addition, the peak orientation in temperature also affects to the polarization pattern. As described in [13], the polarization direction characterizes the flow of the photons. Whilst the polarization direction is radial when the velocity field is converging, it shows a tangential configuration for a divergent flow [18]. In the case of peaks, its shape depends on the correlation between $T$ and polarization. Therefore, in addition to the divergence of the photon flow, the sign of the temperature is also important to describe the polarization pattern. The oscillations in $Q_{r}$ represent changes both in the sign of the temperature and in the velocity field (see [13], for a more detailed explanation). When the peak has eccentricity, it is possible to distinguish two different effects on the velocity field which modify the polarization pattern: a change in the direction of the flow and azimuthal variations of the modulus of the velocity field. Both effects modify the local quadrupole moment of the photon distribution, which causes the CMB polarization. The fact of having a non-spherically symmetric potential makes 
the flow to deviate from being purely radial. This introduces a nonzero $U_{r}$ field, even if the curl contribution is zero (see figure 7). In the principal axes directions, the flow is radial as in the spherical case, and therefore $U_{r}$ vanishes. However, the deviation from the radial flow due to the peak deformation reaches its maximum value in directions at $45^{\circ}$ with respect to the principal axes. For this reason, the azimuthal dependence of $U_{r}$ is a quadrupolar pattern rotated $45^{\circ}$ with respect to the orientation axis. The alternating sign in each quadrupolar lobe indicates that the deviation angle between the velocity field and the radial direction has different signs in each quadrant. In addition, the $U_{r}$ pattern also presents a radial dependence (see figure 6). The changes on the sign in the radial profile is produced by the acoustic oscillations present in the correlation function of the temperature and polarization fields. In addition to the flow direction, the modulus of the velocity field is also affected by the peak eccentricity. In regions where the peak pattern is compressed with respect to the spherical case, the pressure of the photons is higher, and on the contrary, the pressure is lower in the direction of elongation. The pressure of the photon fluid modifies the velocity field, and hence also the polarization pattern. The directions of elongation and compression correspond to the major and minor axes, respectively. This introduces a quadrupolar pattern aligned with the principal axes of the peak, which can be seen in both, $Q_{r}$ and $P$. In some cases, the pressure in the elongation axis is not enough to reverse the flow, and therefore the change of sign in $Q_{r}$ due to the velocity reversion is not present (see figure 5).

In order to enhance the elliptical patterns, the peaks represented in figures 4-8 are selected in the temperature field smoothed with a Gaussian of FWHM $1^{\circ}$, which implies that the inner acoustic oscillations in the $Q_{r}$ profile are suppressed by the filter (compare with figure 3). A calculation of the profiles at high resolution indicates that any source of power at small scales different from the baryon acoustic oscillations (e.g. lensing or noise) produce a smearing of the ring pattern present in the polarization field due to the fact in this situation the peaks do not trace properly the potential wells at the last scattering surface.

\section{Peak simulations}

In this section, we use the formalism developed in section 3 to generate constrained simulations having a peak with given characteristics. For simplicity, we consider the case in which the peak height $\nu$ is fixed to a given value, but it is possible to generalize the procedure for random values of $\nu$. The simulations are generated in the spherical harmonic space. The first step is to generate the variables $\hat{a}_{\ell m}$ defined in eqs. (3.1), which are given as a linear combination of the standard spherical harmonic coefficients $a_{\ell m}$. This property allows us to consider that the $\hat{a}_{\ell m}$ variables are Gaussian under the assumption that the field where the peak is selected is also Gaussian. These new variables obtained after the orthogonalization process are not independent. Their covariance matrix is given by

$$
\begin{gathered}
\left\langle\hat{a}_{\ell 0} \hat{a}_{\ell^{\prime} 0}\right\rangle=\delta_{\ell \ell^{\prime}}+\left(\nu_{\ell} \kappa_{\ell}\right) C^{-1}\left(\begin{array}{c}
\nu_{\ell^{\prime}} \\
\kappa_{\ell^{\prime}}
\end{array}\right) \quad\left(\ell \neq \ell_{\nu}, \ell_{\kappa}\right), \\
\left\langle\hat{a}_{\ell 1} \hat{a}_{\ell^{\prime} 1}\right\rangle=\delta_{\ell \ell^{\prime}}+\frac{\eta_{\ell} \eta_{\ell^{\prime}}}{\eta_{\ell_{\eta}}^{2}} \quad\left(\ell \neq \ell_{\eta}\right), \\
\left\langle\hat{a}_{\ell 2} \hat{a}_{\ell^{\prime} 2}\right\rangle=\delta_{\ell \ell^{\prime}}+\frac{\epsilon_{\ell} \epsilon_{\ell^{\prime}}}{\epsilon_{\ell_{\eta}}^{2}} \quad\left(\ell \neq \ell_{\epsilon}\right), \\
\left\langle\hat{a}_{\ell m} \hat{a}_{\ell^{\prime} m}\right\rangle=\delta_{\ell \ell^{\prime}} \quad(m>2),
\end{gathered}
$$


where $C=P P^{t}$, being $P$ the pivot matrix defined in eq. (3.2). Using the Cholesky decomposition of the covariance matrix, it is possible to simulate the $\hat{a}_{\ell m}$ coefficients.

The next step is to simulate the peak variables. Using the probability in eq. (4.1), we have to put constraints in order to have a minimum or maximum. In practice, the easiest way to do this is by using a Montecarlo approach. Conditioning the peak height to $\nu$, random values of $\kappa$ and $|\epsilon|$ are generated. The eccentricity $\epsilon$ is generated from the two independent Gaussian variables which characterize its real and imaginary parts, while the curvature $\kappa$ is generated as a Gaussian with mean $\rho \nu$ and variance $1-\rho^{2}$ (as it can be deduced from eq. (4.1)). If these numbers satisfy the extremum constraint $|\epsilon| \leq \sqrt{a}|\kappa|$ and $\kappa>0(\kappa<0)$ for maximum (minimum) selection, these values are preserved. Otherwise, they are rejected and generated again until obtaining a pair of values which satisfy the extremum constraint. The sign of $\kappa$ is chosen to be positive or negative depending whether we are selecting minima or maxima respectively.

Once the peak variables $\kappa, \epsilon$ and the $\hat{a}_{\ell m}$ variables are simulated, the standard spherical harmonic coefficients $a_{\ell m}$ are recovered using eqs. (3.3). Given a simulation of the temperature, it is possible to generate the polarization fields $E$ and $B$ correlated with it. In order to do this coherently, we simulate the spherical harmonics coefficients $e_{\ell m}$ and $b_{\ell m}$, which correspond to $E$ and $B$ respectively, following a Gaussian distribution with mean and variance given in eqs. (3.4). The influence of the peak in the polarization fields is given by the correlation between both fields and the temperature.

Notice that although, using this formalism, the peak is located at the north pole, it can be set at any position on the sphere by performing the proper rotation. The last step is to construct the maps from the spherical harmonic coefficients $a_{\ell m}, e_{\ell m}$ and $b_{\ell m}$. A simulation produced following this procedure is given in figure 12 .

\section{Conclusions}

In this paper, the peak statistics and their shape on the sphere is presented. The description of the peaks is given by using the suitable properties of the spherical harmonic space. For this purpose, the peak degrees of freedom are expressed in terms of the spherical harmonics coefficients. The peak variables, and the rest of the degrees of freedom of the field are subject to a decorrelation procedure, allowing an independent treatment of the peak and the rest of the fluctuating random field. In this procedure, the assumption of Gaussianity is essential, since the decorrelation does not guarantee statistical independence for non-Gaussian fields. The different peak shapes are obtained taking the expectation value of the random field, fixing the peak variables to the desired values.

The probability density of the peak variables is also calculated for the sphere. Some differences with respect to previous calculations are found [11], which may be important when the field is dominated by large-scale peaks. The main difference with respect to the flat case is that the variances of the mean curvature $(\kappa)$ and the eccentricity $(\epsilon)$ are not exactly the same. However, these variances are not independent since they are related through a constraint equation (see eq. (B.3)). In the small-scale limit, both variances have the same behaviour (they scale as $\ell^{4}$ ) and the flat approximation is recovered. On the contrary, the variance of the eccentricity is suppressed with respect to the variance of the curvature for large-scale peaks. Therefore, the probability density of $\kappa$ and $\epsilon$ is modified for large peaks on the sphere. However, this effect is only noticeable when the field is dominated by peaks 

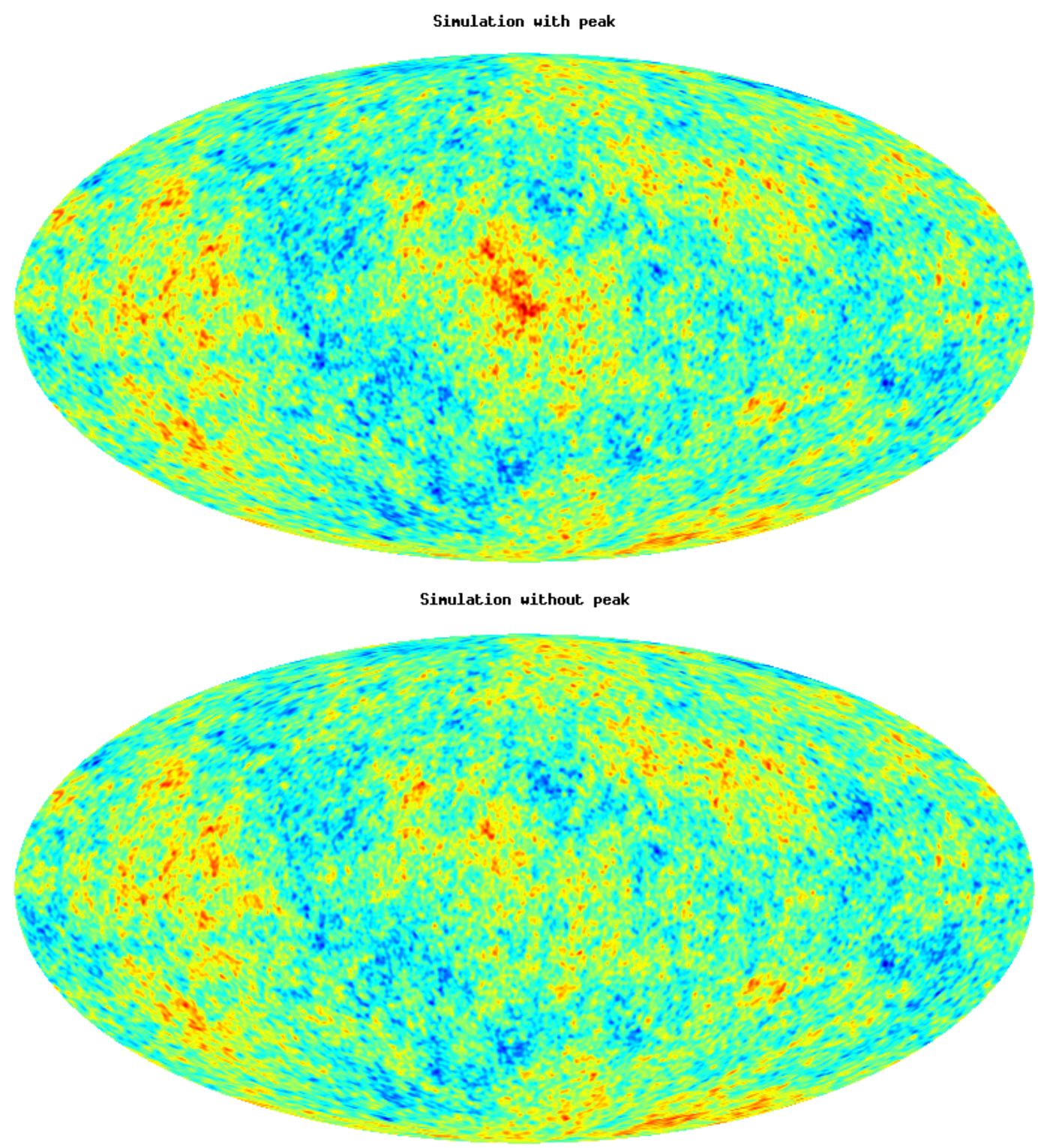

$-5.00$

$+5.00$

Figure 12. Simulations of the CMB temperature field with a peak with $\nu=5$ located at the centre of the image (upper map), and without a peak (lower map). Both simulations only differ in the peak variables (the variables $\hat{a}_{\ell m}$ are the same), and therefore it is possible to see similar structures in regions away from the peak. One can notice that the presence of the peak affects to the area around it, attending to the properties of the temperature correlation function. In these maps, the temperature is filtered with a Gaussian whose FWHM is $1^{\circ}$, and the color bar indicates the value of the map normalized by the standard deviation.

whose size is $\gtrsim 45^{\circ}$. Although these scales are not usually considered, it may be important in the study of the large-scale anomalies.

The peak shape in $T, E$ and $B$ fields for peaks selected in temperature can be understood 
as a biased version of the $T T, T E$ and $T B$ correlation functions respectively. For high peaks, this bias is just a constant. However, when the peak height becomes smaller, the effect of the extremum constraint (minimum or maximum selection) and the peak eccentricity introduce a non-local bias. It is found that this bias is anisotropic due to the eccentricity. In the case that peaks are selected with spherical symmetry, then the non-local isotropic bias is recovered.

Throughout this work, we consider peaks selected in the temperature field allowing nonzero eccentricity. The non-spherical symmetry of peaks introduces a quadrupolar dependence on the azimuthal angle $\phi$, which modifies their local shape. However, this asymmetry only affects to scales smaller than the sound horizon size. For larger scales, the peak shape is only affected by gravity, which is not sensitive to the local eccentricity at the centre. As it is expected, although the peaks are selected in the temperature field, the polarization around the peak location is also affected due to their correlation. The induced shape on the Stokes parameters, and on the $E$ and $B$ polarization fields, has been calculated for the general case of peaks with eccentricity. In the case of the Stokes parameters, we have used the polar coordinates around the peak, leading to the parameters $Q_{r}$ and $U_{r}$ [16]. When peaks have spherical symmetry, and there is no physical effects introducing $T B$ correlation, the induced $U_{r}$ pattern vanishes. However, this is not the case when the peak eccentricity is considered. The asymmetrical photon flow converging or diverging to the potential well introduces a nonzero $U_{r}$ contribution. The shape of $U_{r}$ in this case is a quadrupole whose axes form an angle of $45^{\circ}$ with respect to the peak principal axes. In addition, the Stokes parameter $Q_{r}$ is also modified by the peak eccentricity. The differences in pressure and flow velocity in the directions of elongation and compression of the ellipse introduce a quadrupolar dependence in $Q_{r}$, in this case aligned with the principal axes.

Finally, the peak formalism in the spherical harmonic space developed in this paper allows to generate Gaussian random simulations with a given peak at some position on the sphere. The peak can be chosen with the desired characteristics (peak height, mean curvature and eccentricity). In particular, the extremum constraint can be imposed to the peak variables, generating in this case a minimum or maximum. This mechanism to simulate peaks may be useful for the analysis of particular peaks present in the data, taking into account the possible systematics, noise and mask.

In a future work, we will apply the formalism developed in this paper to CMB data. In particular, we will test the standard cosmological model looking at the curvature and eccentricity of extrema, and considering both temperature and polarization.

\section{A Covariant derivatives on the sphere}

A suitable approach to take derivatives on the sphere is by using the covariant derivatives. The components of a tensor field on the sphere can be expressed in the standard orthonormal basis of the tangent plane, $\mathbf{e}_{\theta}$ and $\mathbf{e}_{\phi}$. For convenience, we change this basis to the helicity basis $\mathbf{e}_{ \pm}=\left(\mathbf{e}_{\theta} \pm i \mathbf{e}_{\phi}\right) / \sqrt{2}$. The interest of working in the helicity basis is that the covariant derivatives can be expressed in terms of the raising and lowering operators $\not \partial$ and $\not^{*}$ :

$$
\nabla_{+}=-\frac{1}{\sqrt{2}} \not \partial, \quad \nabla_{-}=-\frac{1}{\sqrt{2}} \not^{*} .
$$

Throughout the paper, we use $\not \partial$ and $\not{ }^{*}$ as the derivative operators instead of the covariant derivatives, although the difference between both is only a normalization constant. In order 
to differentiate any field on the sphere, it is enough to see how $\not \partial$ and $\not{ }^{*}$ operate over the spin-weighted spherical harmonics:

$$
\begin{gathered}
\not \partial\left({ }_{s} Y_{\ell m}\right)=\sqrt{\ell(\ell+1)-s(s+1)}{ }_{s+1} Y_{\ell m}, \\
\not{ }^{*}\left({ }_{s} Y_{\ell m}\right)=-\sqrt{\ell(\ell+1)-s(s-1)}{ }_{s-1} Y_{\ell m} .
\end{gathered}
$$

For simplicity, we are particularly interested in the value of the derivatives at the north pole. As the spherical coordinates present singularities at both poles, we have to take special care when expressions are evaluated at these points. The problem with the spherical coordinates is that, while $\theta$ takes the values 0 or $\pi$ at the poles, the azimuthal angle $\phi$ is undetermined at these points. Different values of $\phi$ correspond to different orientations of the basis vectors $\mathbf{e}_{\theta}$ and $\mathbf{e}_{\phi}$. Therefore, the value of $\phi$ at the poles characterizes the orientation of the local system of reference. In the case of scalars, the system of reference is not important due to their invariant character. However, for higher order tensors, the orientation modifies their components. In general, if we operate with $\not \partial$ and $\not{ }^{*}$ over the spherical harmonics and evaluate them at the north pole $(\theta=0)$, it is obtained that

$$
\left.(\not)^{*}\right)^{a}(\not \partial)^{b} Y_{\ell m}(0, \phi)=(-1)^{b} \sqrt{\frac{2 \ell+1}{4 \pi}} \sqrt{\frac{(\ell+a-b) !}{(\ell-a+b) !} \frac{(\ell+b) !}{(\ell-b) !}} e^{i(a-b) \phi} \delta_{m, a-b},
$$

where the $\phi$ dependence has been considered. The spinorial character of the derivatives causes that their values are complex numbers. As it is expected, the spin of $\left(\not \partial^{*}\right)^{a}(\not \partial)^{b} Y_{\ell m}$ is $a-b$. This fact is reflected in the exponential factor $e^{i(a-b) \phi}$, which determines its transformation under azimuthal rotations. The presence of $\phi$ in eq. (A.3) is nothing more than an indication of the non-zero spin of the derivatives and the ambiguity of the coordinates at the north pole. For this reason, we can understand the $\phi$ angle in this equation as a gauge parameter, caused by the lack of one-to-one mapping of the spherical coordinates and the sphere. In the following and throughout the calculations in this paper, we use the gauge $\phi=0$ when we evaluate spinorial quantities at the north pole. This corresponds to a particular orientation of the system of reference, aligned with the $x$ and $y$ directions. In this case, we can ignore the factor $e^{i(a-b) \phi}$ in eq. (A.3). In particular, we are interested in some special values of eq. (A.3):

$$
\begin{gathered}
Y_{\ell m}(0,0)=\sqrt{\frac{2 \ell+1}{4 \pi}} \delta_{m 0} \\
\not^{*} Y_{\ell m}(0,0)=\sqrt{\frac{2 \ell+1}{4 \pi}} \sqrt{\frac{(\ell+1) !}{(\ell-1) !}} \delta_{m 1} \\
\not \partial Y_{\ell m}(0,0)=-\sqrt{\frac{2 \ell+1}{4 \pi}} \sqrt{\frac{(\ell+1) !}{(\ell-1) !}} \delta_{m-1} \\
\not \partial^{*} \not \partial Y_{\ell m}(0,0)=-\sqrt{\frac{2 \ell+1}{4 \pi}} \frac{(\ell+1) !}{(\ell-1) !} \delta_{m 0} \\
\left.(\not)^{*}\right)^{2} Y_{\ell m}(0,0)=\sqrt{\frac{2 \ell+1}{4 \pi}} \sqrt{\frac{(\ell+2) !}{(\ell-2) !}} \delta_{m 2} \\
(\not \partial)^{2} Y_{\ell m}(0,0)=\sqrt{\frac{2 \ell+1}{4 \pi}} \sqrt{\frac{(\ell+2) !}{(\ell-2) !}} \delta_{m-2}
\end{gathered}
$$


Finally, in order to calculate the Stokes parameters, it is useful to obtain the expressions for the 2-spin spherical harmonics, in particular for $m=0$ and $m=2:^{3}$

$$
\begin{aligned}
& { }_{ \pm 2} Y_{\ell 0}(\theta, \phi)=Y_{\ell \pm 2}(\theta, \phi) e^{\mp i 2 \phi}=\sqrt{\frac{2 \ell+1}{4 \pi}} \sqrt{\frac{(\ell-2) !}{(\ell+2) !}} P_{\ell}^{2}(\cos \theta), \\
& { }_{ \pm 2} Y_{\ell 2}(\theta, \phi)=2 \sqrt{\frac{2 \ell+1}{4 \pi}} \frac{(\ell-2) !}{(\ell+2) !}\left(P_{\ell}^{+}(\cos \theta) \pm P_{\ell}^{-}(\cos \theta)\right) e^{i 2 \phi},
\end{aligned}
$$

where the functions defined in eqs. (5.7) were used.

\section{B Peak degrees of freedom}

In this appendix, we study the peak degrees of freedom and their connection to the operators defined in the previous appendix. Peaks are described by derivatives up to second order. Assuming that the field is given by its spherical harmonic expansion (see eq. (2.1)), and that the peak is located at the north pole, only the $m=0$ spherical harmonic coefficients contribute to the value of $\nu$ (eq. (2.4a)). However, the first derivatives of $T$ at the north pole are given by the real and imaginary parts of $\not^{*} T$, which is a linear combination of the spherical harmonics coefficients with $m=1$ (eq. (2.2b)). The second order derivatives are encoded in the Hessian matrix, which can be written in the following way:

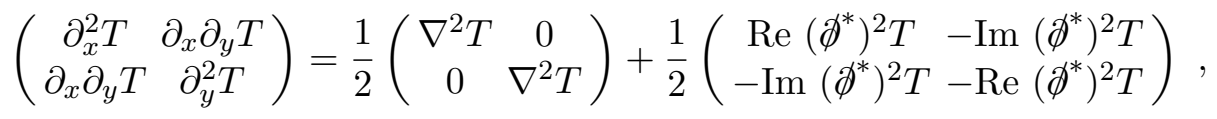

where $\nabla^{2} T$ is the Laplacian corresponding to the trace of the Hessian matrix. It can be written in terms of the operators described in the previous appendix: $\nabla^{2} T=\not \not^{*} \not \partial T$. The complex number $\left(\not \partial^{*}\right)^{2} T$ is the traceless part and it describes the eccentricity of the peak. The Hessian matrix is separated in this form because the two parts transform in a different way. The Laplacian is invariant under rotations around the origin, while the $\left(\not \not^{*}\right)^{2} T$ transforms like a spin-2 tensor. The physical meaning of the Laplacian is the mean curvature of the peak when it is averaged over all directions. Whilst the modulus of $\left(\not \not^{*}\right)^{2} T$ is proportional to the square of the eccentricity of the peak, the orientation angle is encoded in its phase.

Throughout this paper, the peak variables $\nu, \kappa, \eta$ and $\epsilon$ are used. They are defined as the quantities $T,-\nabla^{2} T, \not^{*} T$ and $\left(\not^{*}\right)^{2} T$, normalized to unit variance. The variances of the peak variables are

$$
\begin{gathered}
\sigma_{\nu}^{2}=\left\langle T^{2}\right\rangle=\sum_{\ell=0}^{\infty} \frac{2 \ell+1}{4 \pi} C_{\ell}^{T T}, \\
\sigma_{\kappa}^{2}=\left\langle\left(-\nabla^{2} T\right)^{2}\right\rangle=\sum_{\ell=0}^{\infty} \frac{2 \ell+1}{4 \pi}\left[\frac{(\ell+1) !}{(\ell-1) !}\right]^{2} C_{\ell}^{T T}, \\
\sigma_{\eta}^{2}=\left\langle\left|\partial^{*} T\right|^{2}\right\rangle=\sum_{\ell=1}^{\infty} \frac{2 \ell+1}{4 \pi} \frac{(\ell+1) !}{(\ell-1) !} C_{\ell}^{T T}, \\
\sigma_{\epsilon}^{2}=\left\langle\left|\left(\not \partial^{*}\right)^{2} T\right|^{2}\right\rangle=\sum_{\ell=2}^{\infty} \frac{2 \ell+1}{4 \pi} \frac{(\ell+2) !}{(\ell-2) !} C_{\ell}^{T T} .
\end{gathered}
$$

\footnotetext{
${ }^{3}$ The spherical harmonics for $m=-2$ are calculated using the property ${ }_{ \pm s} Y_{\ell-m}=(-1)^{m+s}{ }_{\mp s} Y_{\ell m}^{*}$.
} 
In previous works [11, 13], it was implicitly assumed that $\sigma_{\kappa}^{2}$ and $\sigma_{\epsilon}^{2}$ are equal, but we show that they are different if the exact calculation on the sphere is done. In particular, both variances have a $\ell^{4}$ behavior at small scales $(\ell \gg 1)$ and thus they tend to be equal. On the contrary, if the field is dominated by large-scale fluctuations, the variances $\sigma_{\kappa}^{2}$ and $\sigma_{\epsilon}^{2}$ are different and this has an effect on the peak statistics. In addition, these two variances are not independent, as they are related through the following equation:

$$
\sigma_{\kappa}^{2}-\sigma_{\epsilon}^{2}=2 \sigma_{\eta}^{2}
$$

In the limit when $\sigma_{\kappa}^{2}, \sigma_{\epsilon}^{2} \gg \sigma_{\eta}^{2}$ it is possible to consider that the variances $\sigma_{\kappa}^{2}$ and $\sigma_{\epsilon}^{2}$ are equal. The peak variables $\nu, \kappa, \eta$ and $\epsilon$ are obtained normalizing by the respective variance. In this situation, the multipolar coefficients of the peak variables are

$$
\begin{gathered}
\nu_{\ell}=\sqrt{\frac{2 \ell+1}{4 \pi} \frac{C_{\ell}^{T T}}{\sigma_{\nu}^{2}}}, \\
\kappa_{\ell}=\sqrt{\frac{2 \ell+1}{4 \pi} \frac{C_{\ell}^{T T}}{\sigma_{\kappa}^{2}} \frac{(\ell+1) !}{(\ell-1) !}}, \\
\eta_{\ell}=\sqrt{\frac{2 \ell+1}{4 \pi} \frac{C_{\ell}^{T T}}{\sigma_{\eta}^{2}}} \sqrt{\frac{(\ell+1) !}{(\ell-1) !}}, \\
\epsilon_{\ell}=\sqrt{\frac{2 \ell+1}{4 \pi} \frac{C_{\ell}^{T T}}{\sigma_{\epsilon}^{2}}} \sqrt{\frac{(\ell+2) !}{(\ell-2) !}},
\end{gathered}
$$

where the factor $C_{\ell}^{T T}$ has been introduced in order to have normalized $a_{\ell m}$ coefficients. It is useful to calculate the covariance of the peak height, the mean curvature, the first derivative and the eccentricity. The covariance of $\nu, \kappa, \eta$ and $\epsilon$ is: ${ }^{4}$

$$
S=\left(\begin{array}{llll}
1 & \rho & 0 & 0 \\
\rho & 1 & 0 & 0 \\
0 & 0 & 1 & 0 \\
0 & 0 & 0 & 1
\end{array}\right)
$$

where $\rho=\sigma_{\eta}^{2} / \sigma_{\nu} \sigma_{\kappa}$ is the correlation between $\nu$ and $\kappa$. We also consider the covariance of the scalar degrees of freedom $\nu$ and $\kappa$, which is a submatrix of $S$ :

$$
\Sigma=\left(\begin{array}{ll}
1 & \rho \\
\rho & 1
\end{array}\right)
$$

\section{Flat approximation}

In this appendix we see how to calculate the small-angle limit of the expressions developed throughout this paper. In particular, the expressions given in [13] are recovered. The dictionary between the sphere and the flat approximation is given by

$$
(-1)^{m} \sqrt{\frac{(\ell-m) !}{(\ell+m) !}} P_{\ell}^{m}(\cos \theta) \quad \longrightarrow \quad J_{m}(\ell \theta),
$$

\footnotetext{
${ }^{4}$ The covariance of complex variables $\left\{x_{i}\right\}$ is defined as $\left\langle x_{i}^{*} x_{j}\right\rangle-\left\langle x_{i}^{*}\right\rangle\left\langle x_{j}\right\rangle$.
} 


$$
\begin{aligned}
\sum_{\ell=0}^{\infty} \frac{2 \ell+1}{4 \pi} & \longrightarrow \int \frac{\mathrm{d} \ell}{\ell} \frac{\ell(\ell+1)}{2 \pi}, \\
\ell(\ell+1) & \longrightarrow \ell^{2} .
\end{aligned}
$$

These transformations are valid for large multipoles $(\ell \gg 1)$ and small angles $(\theta \ll 1)$ such that $\ell \theta \sim 1$. In this case, the associated Legendre functions $P_{\ell}^{m}(\cos \theta)$ are replaced by the Bessel function $J_{m}(\ell \theta)$ of order $m$. This relation can be deduced applying the small-angle limit to the fundamental equation of the Legendre functions. The sums over multipoles are replaced by an integral over $\ell$ with the appropriate volume factor.

\section{Acknowledgments}

Partial financial support from the Spanish Ministerio de Economía y Competitividad Projects AYA2012-39475-C02-01 and Consolider-Ingenio 2010 CSD2010-00064 is acknowledged.

\section{References}

[1] Planck Collaboration, N. Aghanim, M. Arnaud, M. Ashdown, J. Aumont, C. Baccigalupi, A. J. Banday, R. B. Barreiro, J. G. Bartlett, N. Bartolo, and et al., Planck 2015 results. XI. CMB power spectra, likelihoods, and robustness of parameters, ArXiv e-prints (July, 2015) [arXiv: 1507.02704].

[2] D. Hanson, S. Hoover, A. Crites, P. A. R. Ade, K. A. Aird, J. E. Austermann, J. A. Beall, A. N. Bender, B. A. Benson, L. E. Bleem, J. J. Bock, J. E. Carlstrom, C. L. Chang, H. C. Chiang, H.-M. Cho, A. Conley, T. M. Crawford, T. de Haan, M. A. Dobbs, W. Everett, J. Gallicchio, J. Gao, E. M. George, N. W. Halverson, N. Harrington, J. W. Henning, G. C. Hilton, G. P. Holder, W. L. Holzapfel, J. D. Hrubes, N. Huang, J. Hubmayr, K. D. Irwin, R. Keisler, L. Knox, A. T. Lee, E. Leitch, D. Li, C. Liang, D. Luong-Van, G. Marsden, J. J. McMahon, J. Mehl, S. S. Meyer, L. Mocanu, T. E. Montroy, T. Natoli, J. P. Nibarger, V. Novosad, S. Padin, C. Pryke, C. L. Reichardt, J. E. Ruhl, B. R. Saliwanchik, J. T. Sayre, K. K. Schaffer, B. Schulz, G. Smecher, A. A. Stark, K. T. Story, C. Tucker, K. Vanderlinde, J. D. Vieira, M. P. Viero, G. Wang, V. Yefremenko, O. Zahn, and M. Zemcov, Detection of B-Mode Polarization in the Cosmic Microwave Background with Data from the South Pole Telescope, Physical Review Letters 111 (Oct., 2013) 141301, [arXiv:1307.5830].

[3] The Polarbear Collaboration: P. A. R. Ade, Y. Akiba, A. E. Anthony, K. Arnold, M. Atlas, D. Barron, D. Boettger, J. Borrill, S. Chapman, Y. Chinone, M. Dobbs, T. Elleflot, J. Errard, G. Fabbian, C. Feng, D. Flanigan, A. Gilbert, W. Grainger, N. W. Halverson, M. Hasegawa, K. Hattori, M. Hazumi, W. L. Holzapfel, Y. Hori, J. Howard, P. Hyland, Y. Inoue, G. C. Jaehnig, A. H. Jaffe, B. Keating, Z. Kermish, R. Keskitalo, T. Kisner, M. Le Jeune, A. T. Lee, E. M. Leitch, E. Linder, M. Lungu, F. Matsuda, T. Matsumura, X. Meng, N. J. Miller, H. Morii, S. Moyerman, M. J. Myers, M. Navaroli, H. Nishino, A. Orlando, H. Paar, J. Peloton, D. Poletti, E. Quealy, G. Rebeiz, C. L. Reichardt, P. L. Richards, C. Ross, I. Schanning, D. E. Schenck, B. D. Sherwin, A. Shimizu, C. Shimmin, M. Shimon, P. Siritanasak, G. Smecher, H. Spieler, N. Stebor, B. Steinbach, R. Stompor, A. Suzuki, S. Takakura, T. Tomaru, B. Wilson, A. Yadav, and O. Zahn, A Measurement of the Cosmic Microwave Background B-mode Polarization Power Spectrum at Sub-degree Scales with POLARBEAR, ApJ 794 (Oct., 2014) 171, [arXiv:1403.2369].

[4] BICEP2/Keck and Planck Collaborations, P. A. R. Ade, N. Aghanim, Z. Ahmed, R. W. Aikin, K. D. Alexander, M. Arnaud, J. Aumont, C. Baccigalupi, A. J. Banday, and et al., Joint Analysis of BICEP2/Keck Array and Planck Data, Physical Review Letters 114 (Mar., 2015) 101301, [arXiv: 1502.00612]. 
[5] BICEP2 and Keck Array Collaborations, P. A. R. Ade, Z. Ahmed, R. W. Aikin, K. D. Alexander, D. Barkats, S. J. Benton, C. A. Bischoff, J. J. Bock, J. A. Brevik, I. Buder, E. Bullock, V. Buza, J. Connors, B. P. Crill, C. D. Dowell, C. Dvorkin, L. Duband, J. P. Filippini, S. Fliescher, S. R. Golwala, M. Halpern, S. Harrison, M. Hasselfield, S. R. Hildebrandt, G. C. Hilton, V. V. Hristov, H. Hui, K. D. Irwin, K. S. Karkare, J. P. Kaufman, B. G. Keating, S. Kefeli, S. A. Kernasovskiy, J. M. Kovac, C. L. Kuo, E. M. Leitch, M. Lueker, P. Mason, K. G. Megerian, C. B. Netterfield, H. T. Nguyen, R. O'Brient, R. W. Ogburn, IV, A. Orlando, C. Pryke, C. D. Reintsema, S. Richter, R. Schwarz, C. D. Sheehy, Z. K. Staniszewski, R. V. Sudiwala, G. P. Teply, K. L. Thompson, J. E. Tolan, A. D. Turner, A. G. Vieregg, A. C. Weber, J. Willmert, C. L. Wong, and K. W. Yoon, BICEP2/Keck Array V: Measurements of B-mode Polarization at Degree Angular Scales and $150 \mathrm{GHz}$ by the Keck Array, ApJ 811 (Oct., 2015) 126, [arXiv:1502.00643].

[6] Planck Collaboration, P. A. R. Ade, N. Aghanim, M. Arnaud, M. Ashdown, J. Aumont, C. Baccigalupi, A. J. Banday, R. B. Barreiro, J. G. Bartlett, and et al., Planck 2015 results. XIII. Cosmological parameters, ArXiv e-prints (Feb., 2015) [arXiv:1502.01589].

[7] Planck Collaboration, P. A. R. Ade, N. Aghanim, Y. Akrami, P. K. Aluri, M. Arnaud, M. Ashdown, J. Aumont, C. Baccigalupi, A. J. Banday, and et al., Planck 2015 results. XVI. Isotropy and statistics of the CMB, ArXiv e-prints (June, 2015) [arXiv:1506.07135].

[8] J. M. Bardeen, J. R. Bond, N. Kaiser, and A. S. Szalay, The statistics of peaks of Gaussian random fields, ApJ 304 (May, 1986) 15-61.

[9] V. Desjacques, Local bias approach to the clustering of discrete density peaks, Phys. Rev. D 87 (Feb., 2013) 043505, [arXiv: 1211.4128].

[10] V. Desjacques, Baryon acoustic signature in the clustering of density maxima, Phys. Rev. D 78 (Nov., 2008) 103503, [arXiv:0806.0007].

[11] J. R. Bond and G. Efstathiou, The statistics of cosmic background radiation fluctuations, MNRAS 226 (June, 1987) 655-687.

[12] R. B. Barreiro, J. L. Sanz, E. Martínez-González, L. Cayón, and J. Silk, Peaks in the Cosmic Microwave Background: Flat versus Open Models, ApJ 478 (Mar., 1997) 1-6, [astro-ph/9612114].

[13] E. Komatsu, K. M. Smith, J. Dunkley, C. L. Bennett, B. Gold, G. Hinshaw, N. Jarosik, D. Larson, M. R. Nolta, L. Page, D. N. Spergel, M. Halpern, R. S. Hill, A. Kogut, M. Limon, S. S. Meyer, N. Odegard, G. S. Tucker, J. L. Weiland, E. Wollack, and E. L. Wright, Seven-year Wilkinson Microwave Anisotropy Probe (WMAP) Observations: Cosmological Interpretation, ApJS 192 (Feb., 2011) 18, [arXiv:1001.4538].

[14] C. R. Contaldi, Imaging parity-violation in the CMB, ArXiv e-prints (Oct., 2015) [arXiv: 1510.02629].

[15] M. Galaverni, G. Gubitosi, F. Paci, and F. Finelli, Cosmological birefringence constraints from CMB and astrophysical polarization data, J. Cosmology Astropart. Phys. 8 (Aug., 2015) 31, [arXiv: 1411.6287].

[16] M. Kamionkowski, A. Kosowsky, and A. Stebbins, Statistics of cosmic microwave background polarization, Phys. Rev. D 55 (June, 1997) 7368-7388, [astro-ph/9611125].

[17] A. Stebbins, Weak Lensing On the Celestial Sphere, ArXiv Astrophysics e-prints (Sept., 1996) [astro-ph/9609149].

[18] D. Coulson, R. G. Crittenden, and N. G. Turok, Polarization and anisotropy of the microwave sky, Physical Review Letters 73 (Oct., 1994) 2390-2393, [astro-ph/9406046]. 